\title{
Higher covariant derivative Pauli-Villars regularization does not lead to a consistent QCD
}

\author{
C.P. Martin ${ }^{\mathrm{a}}$, F. Ruiz Ruiz ${ }^{\mathrm{b}}$ \\ a Departamento de Física Teórica, C-XI, Universidad Autónoma de Madrid, Cantoblanco, \\ Madrid 28049, Spain \\ b NIKHEF-H, Postbus 41882, 1009 DB Amsterdam, The Netherlands
}

Received 2 September 1994; accepted 17 November 1994

\begin{abstract}
We compute the beta function at one loop for Yang-Mills theory using as regulator the combination of higher covariant derivatives and Pauli-Villars determinants proposed by Faddeev and Slavnov. This regularization prescription has the appealing feature that it is manifestly gauge invariant and essentially four-dimensional. It happens however that the one-loop coefficient in the beta function that it yields is not $-11 / 3$, as it should be, but $-23 / 6$. The difference is due to unphysical logarithmic radiative corrections generated by the Pauli-Villars determinants on which the regularization method is based. This no-go result discards the prescription as a viable gauge invariant regularization, thus solving a long-standing open question in the literature. We also observe that the prescription can be modified so as to not generate unphysical logarithmic corrections, but at the expense of losing manifest gauge invariance.
\end{abstract}

\section{Introduction}

To regularize and renormalize a theory with symmetries it is very convenient to use a regularization method and a renormalization scheme that preserve the symmetries of the theory. In the case of gauge theories, there are not so many regularization methods that preserve gauge invariance. The two traditional candidates are dimensional regularization [1] and higher covariant derivatives [2,3] - more recent regularization methods [4,5] will not concern us here. Dimensional regularization works well for vector gauge theories like Yang-Mills or QCD but presents some problems when it comes to dealing with theories with $\gamma^{5}$. As regards higher covariant derivative regularization, it has the appealing feature that it does not have problems with $\gamma^{5}$, thus constituting an in principle useful tool to study anomalies. Furthermore, there are suggestions [6] that 
it leads to supersymmetric as well as gauge invariant regularization procedures. Yet it is not known whether higher covariant derivative regulators work even for the simplest cases, like ordinary Yang-Mills or QCD. In this paper we provide an answer to this question in the negative for the case of Yang-Mills theory.

So let us consider Yang-Mills theory. Higher covariant derivative regularization as proposed in Ref. [3] combines a higher covariant derivative term $\left(D^{2} F\right)^{2} / 4 \Lambda^{4}$ with a set of gauge invariant Pauli-Villars determinants. There is some controversy in the literature as to whether or not this prescription works. On the one side there is the position defended in Ref. [3], that claims that the prescription works to all orders in perturbation theory without problems. On the other there is the position of Ref. [7] that states that the prescription only regularizes one-loop divergences and leaves two and higher loops unregularized. Even worse, Ref. [8] sustains that already at one loop the divergences that arise in the regularized Green functions when the regulators are removed can not be subtracted in a gauge invariant fashion, so that the resulting renormalized theory is not gauge invariant. This conclusion is in turn somewhat in contradiction with the non-multiplicative renormalization schemes discussed in Ref. [9]. The problem is that many of these claims, if not all, are based on rather qualitative arguments and are not supported by explicit calculations, thus making it difficult to conclude about the viability of the prescription. To the best of our knowledge, nowhere in the literature physical issues like unitarity or whether the prescription reproduces asymptotic freedom with the correct beta function are addressed.

In this paper we use the prescription to explicitly regularize and renormalize YangMills theory at one loop and to compute the beta function at this order in perturbation theory. It turns out that the renormalized theory is gauge invariant, thus giving lie to Ref. [8], but it also turns out that the Pauli-Villars determinants generate unphysical logarithmic radiative corrections. These unphysical corrections modify the beta function of the theory so that the one-loop coefficient in the beta function comes out to be $-23 / 6$ and not $-11 / 3$, as it should. In Minkowski space these extra corrections change the imaginary part of some 1PI Green functions. This is in contradiction with general results from renormalization theory according to which the one-loop imaginary part of a 1PI Green function is independent of the renormalization procedure. All this implies that the resulting renormalized theory is inconsistent and that the prescription is not an acceptable one and must be disregarded.

The paper is organized as follows. In Section 2 we review the regularization prescription. We spend some time on this since there are a few issues which have passed unnoticed in the previous literature and they play a crucial part in the outcome of this investigation. In Section 3 we show that it is possible to subtract the divergences from the regularized theory and have at the same time gauge invariance. In Section 4 we compute the beta function at one loop and obtain that it does not have the correct coefficient. Section 5 is devoted to prove that the source of the unwanted contributions to the beta function is the Pauli-Villars determinants on which the prescription is based. We include two appendices which contain the Feynman rules (Appendix A) and calculational details (Appendix B). 


\section{The regularization method}

Let us start introducing our notation and conventions. The theory we are interested in is Yang-Mills theory in four-dimensional Euclidean space. The classical action in a general covariant gauge has the form

$$
S=S_{\mathrm{YM}}+S_{\mathrm{GF}},
$$

where $S_{\mathrm{YM}}$ and $S_{\mathrm{GF}}$ are given by

$$
S_{\mathrm{YM}}=\frac{1}{4} \int \mathrm{d}^{4} x F_{\mu \nu}^{a} F^{a \mu \nu}
$$

and

$$
S_{\mathrm{GF}}=\int \mathrm{d}^{4} x\left[\frac{\alpha}{2} b^{a} b^{a}-b^{a}\left(\partial_{\mu} A^{a \mu}\right)+\bar{c}^{a}\left(\partial_{\mu} D^{\mu} c\right)^{a}\right] .
$$

Here $F_{\mu \nu}^{a}=\partial_{\mu} A_{\nu}^{a}-\partial_{\nu} A_{\mu}^{a}+g f^{a b c} A_{\mu}^{b} A_{\nu}^{c}$, denotes the field strength, $A_{\mu}^{a}$ the gauge field, $g$ the coupling constant and $f^{a b c}$ the structure constants of the gauge algebra. The parameter $\alpha$ is the gauge-fixing parameter, $b^{a}$ is the auxiliary field, $\bar{c}^{a}$ and $c^{a}$ are the Faddeev-Popov ghosts and $D_{\mu}^{a c}=\delta^{a c}+g f^{a b c} A_{\mu}^{b}$ is the covariant derivative. The Landau gauge corresponds to $\alpha=0$, and the Feynman gauge to $\alpha=1$. We will assume that the gauge group is a compact, simple Lie group so that the structure constants $f^{a b c}$ can be taken completely antisymmetric in their indices without loss of generality. Furthermore, we will normalize $f^{a b c}$ so that $f^{a c d} f^{b c d}=c_{V} \delta^{a b}$, with $c_{V}$ the quadratic Casimir operator in the adjoint representation $\left[c_{V}=N\right.$ for a gauge group $\left.\mathrm{SU}(N)\right]$. After gauge fixing, gauge invariance takes the form of BRS invariance and so the symmetry to keep under control is the BRS symmetry. The BRS transformations read in our notation

$$
s A_{\mu}^{a}=\left(D_{\mu} c\right)^{a}, \quad s b^{a}=0 \quad s \bar{c}^{a}=b^{a}, \quad s c^{a}=-\frac{1}{2} g f^{a b c} c^{b} c^{c}
$$

with the BRS operator $s$ satisfying as usual $s^{2}=0$. Finally, the generating functional is formally given by

$$
Z[J, \chi, \eta, \bar{\eta}]=\int \mathcal{D} A \mathcal{D} b \mathcal{D} \bar{c} \mathcal{D} c \mathrm{e}^{-\left(S+S_{J}\right)},
$$

where $S$ is as in Eqs. (2.1)-(2.3), $S_{J}$ is the source term

$$
S_{J}=\int \mathrm{d}^{4} x\left(J^{a \mu} A_{\mu}^{a}+\chi^{a} b^{a}+\bar{c}^{a} \eta^{a}+\bar{\eta}^{a} c^{a}\right)
$$

and $J^{a \mu}, \chi^{a}, \eta^{a}$ and $\bar{\eta}^{a}$ are sources for the fields $A_{\mu}^{a}, b^{a}, \bar{c}^{a}$ and $c^{a}$, respectively.

Since it will be used as a point of reference below, let us recall how power counting goes. Given a 1 PI Feynman diagram with $E_{A}$ external gauge lines, $E_{g}$ external ghost lines and $L$ loops, its overall UV degree $\omega$ is given by

$$
\omega=4-E_{A}-\frac{3}{2} E_{g} .
$$


Superficially divergent 1PI diagrams have $\omega \geqslant 0$ and correspond to Green functions already present in the classical action $S$. As is well known, this ensures renormalizability. To actually renormalize the theory, one proceeds in two steps. The first one is to provide a regularization method that renders all Green functions finite order by order in perturbation theory. The second one is to provide a renormalization scheme that eliminates the divergences appearing in the Green functions when the regulator is removed. In this paper we use higher covariant derivative Pauli-Villars regularization as proposed by Faddeev and Slavnov [2,3] to explicitly renormalize Yang-Mills theory at one loop. As announced in the introduction, it will turn out that the resulting renormalized theory is inconsistent. In the course of our investigation we have found that there are a few issues concerning the regularization method itself that have gone unnoticed in the previous literature and that play a crucial role in the result. We thus find worth spending some time in reviewing the method and bringing these points to light.

\subsection{Higher covariant derivative regulators}

The method proposed by Faddeev and Slavnov regularizes Yang-Mills theory in two steps. In the first one, it introduces a higher covariant derivative mass $A$ by replacing the classical gauge-fixed action $S$ in Eq. (2.1) with the action [3]

$$
S_{A}=S_{\mathrm{YM}}+S_{2}+S_{\mathrm{GF}}^{f},
$$

where $S_{\mathrm{YM}}$ is as in Eq. (2.2) and $S_{2}$ and $S_{\mathrm{GF}}^{f}$ are respectively given by

$$
S_{2}=\frac{1}{4 \Lambda^{4}} \int \mathrm{d}^{4} x\left(D^{2} F_{\mu \nu}\right)^{a}\left(D^{2} F^{\mu \nu}\right)^{a}
$$

and

$$
S_{\mathrm{GF}}^{f}=\int \mathrm{d}^{4} x\left[\frac{\alpha}{2} b^{a} \frac{1}{f^{2}\left(\partial^{2} / A^{2}\right)} b^{a}-b^{a}\left(\partial_{\mu} A^{a \mu}\right)+\bar{c}^{a}\left(\partial_{\mu} D^{\mu} c\right)^{a}\right] .
$$

Here $f\left(\partial^{2} / \Lambda^{2}\right)$ is a polynomial in its argument that in momentum space becomes a function of $p^{2} / \Lambda^{2}$ and that by definition is chosen to satisfy the conditions

$$
\begin{aligned}
& f\left(p^{2} / \Lambda^{2}\right) \rightarrow 1 \quad \text { as } \Lambda \rightarrow \infty \\
& f\left(p^{2} / \Lambda^{2}\right) \sim\left(p^{2} / \Lambda^{2}\right)^{\gamma} \quad \text { as } \quad|p| \rightarrow \infty,
\end{aligned}
$$

with $\gamma \geqslant 1$. In this paper we will take

$$
f\left(\frac{p^{2}}{\Lambda^{2}}\right)=1+\frac{p^{4}}{\Lambda^{4}}
$$

for reasons that we explain below. The propagator of the gauge field for the action $S_{A}$ has the form

$$
D_{\mu \nu}^{a b}(p)=\delta^{a b}\left[\frac{\Lambda^{4}}{p^{4}\left(p^{4}+\Lambda^{4}\right)}\left(p^{2} g_{\mu \nu}-p_{\mu} p_{\nu}\right)+\alpha \frac{p_{\mu} p_{\nu}}{p^{4} f^{2}\left(p^{2} / \Lambda^{2}\right)}\right],
$$


where $p^{2 n}$ is a shorthand notation for $\left(p^{2}\right)^{n}$. The second condition in Eq. (2.8) ensures that the propagator goes as $1 / p^{6}$ when $p^{\mu} \rightarrow \infty$. This and some power counting implies that the overall UV degree $\omega_{A}$ of a 1PI diagram generated by $S_{A}$ with $E_{A}$ external gauge legs, $E_{g}$ external ghost legs and $L$ loops is given by [compare with Eq. (2.5)]

$$
\omega_{A}=4-4(L-1)-E_{A}-\frac{7}{2} E_{g}
$$

Now only one-loop 1PI diagrams with $E_{A}=2,3,4$ and $E_{g}=0$ are superficially divergent. We thus see that the higher covariant derivative regulator $\Lambda$ improves the power counting behaviour of the theory but does not completely regularize it. To achieve a complete regularization, one must regularize the one-loop diagrams for which $\omega_{A} \geqslant 0$. Before doing so, let us make a few comments that we feel are in order.

Comment 1. There are many possible choices for $f$ compatible with conditions (2.8). The choice in Eq. (2.9) ensures that $\alpha$-dependent contributions in any 1PI diagram are finite by power counting, as can be trivially checked. In fact, any $f$ for which $\gamma$ in Eq. (2.8) is larger or equal than 2 leads to superficially convergent $\alpha$-dependent contributions. We have taken $f$ as in Eq. (2.9) since in this way the denominator of the propagator $D_{\mu \nu}^{a b}(p)$ has factors only of type $p^{4}$ and $p^{4}+A^{4}$. Note also that we have required $f$ to be polynomial so as to ensure locality.

Comment 2. Since the action $S_{\lambda}$ is BRS invariant, the higher covariant derivative regulator $\Lambda$ preserves BRS invariance.

Comment 3. Note that all 1PI diagrams generated by $S_{\Lambda}$ with external ghost legs are finite by power counting. This implies that Yang-Mills 1PI diagrams with external ghosts are fully regularized by the higher covariant derivative regulator $\Lambda$.

Comment 4 . One may wonder whether the theory can be completely regularized by means of a higher covariant derivative term of higher order in the covariant derivative. The answer is no. To see this, consider the term

$$
S_{n}=\frac{(-)^{n}}{4 \Lambda^{2 n}} \int \mathrm{d}^{4} x F_{\mu \nu}^{a}\left(D^{2 n} F^{\mu \nu}\right)^{a},
$$

where $n$ is an arbitrary positive integer. Power counting now implies that

$$
\omega_{A}(n)=4-2 n(L-1)-E_{A}-\left(\frac{3}{2}+n\right) g .
$$

So no matter how large $n$ is, $\omega_{A}(n)$ is always negative for $L=1, E_{g}=0$ and $E_{A}=2,3,4$.

\subsection{Pauli-Villars determinants}

To regularize the one-loop divergences in the Feynman diagram expansion generated by the action $S_{A}$ above, one can proceed as follows [2,3]. Consider the two following generating functionals labeled by an index $r=0,1$ :

$Z_{r}[J, \chi, \eta, \bar{\eta}]=\int \mathcal{D} A \mathcal{D} b \mathcal{D} \bar{c} \mathcal{D} c \mathrm{e}^{-\left(S_{A}+S_{J}\right)} \prod_{j=1}^{J}\left(\operatorname{det} \mathbb{A}_{j}^{r}\right)^{-\alpha_{j} / 2} \prod_{i=1}^{I}\left(\operatorname{det} \mathbb{C}_{i}^{r}\right)^{\gamma_{i}}$ 
Here $\left\{\alpha_{j}\right\}_{J}$ and $\left\{\gamma_{i}\right\}_{I}$ are real parameters to be fixed and $\operatorname{det} \mathbb{A}_{j}^{r}$, and $\operatorname{det} \mathbb{C}_{i}^{r}$, are defined by

$$
\left(\operatorname{det} \mathbb{A}_{j}^{r}\right)^{-1 / 2}=\int \mathcal{D} A_{j} \mathcal{D} b_{j} \exp \left\{-\left(S_{M_{j}}+S_{b_{j}}\right)\right\}
$$

and

$$
\operatorname{det} \mathbb{C}_{i}^{r}=\int \mathcal{D} \bar{c}_{j} \mathcal{D} c_{j} \exp \left\{-S_{m_{i}}\right\},
$$

with

$$
\begin{aligned}
S_{M_{j}} & =\frac{1}{2} \int \mathrm{d}^{4} x \int \mathrm{d}^{4} y A_{j \mu}^{a}(x)\left[\frac{\delta^{2} S_{A}}{\delta A_{\mu}^{a}(x) \delta A_{\nu}^{b}(y)}-M_{j}^{2} \delta^{a b} g^{\mu \nu} \delta(x-y)\right] A_{j \nu}^{b}(y), \\
S_{b_{j}} & =-\int \mathrm{d}^{4} x b_{j}^{a}\left(\delta^{a b} \partial_{\mu}+r g f^{a c b} A_{\mu}^{c}\right) A_{j}^{b \mu}, \\
S_{m_{i}} & =\int \mathrm{d}^{4} x\left[\bar{c}_{i}^{a}\left(\partial_{\mu} D^{\mu} c_{i}\right)^{a}+r g f^{a b c} \bar{c}_{i}^{a} A_{\mu}^{b}\left(D^{\mu} c_{i}\right)^{c}-m_{i}^{2} \bar{c}_{i}^{a} c_{i}^{a}\right] .
\end{aligned}
$$

The fields $\left\{A_{j \mu}^{a}\right\}$ are commuting Pauli-Villars fields of mass $\left\{M_{j}\right\}$, the fields $b_{j}^{a}$ are Pauli-Villars Lagrange multipliers imposing the condition

$$
\left(\delta^{a b} \partial_{\mu}+\operatorname{rg} f^{a c b} A_{\mu}^{c}\right) A_{j}^{b \mu}=0
$$

and the fields $\left\{\bar{c}_{i}^{a}, c_{i}^{a}\right\}$ are anticommuting Pauli-Villars fields of mass $\left\{m_{i}\right\}$. The determinants $\operatorname{det} \mathbb{A}_{j}^{r}$ and $\operatorname{det} \mathbb{C}_{i}^{r}$ are BRS invariant if $r=1$-actually gauge invariant since they do not depend on ghosts. This follows from the observation [3] that the change of integration variables $\phi \rightarrow \phi+\theta(s \phi)$, where $\theta$ is an anticommuting parameter and $s \phi$ is given by

$$
\begin{aligned}
s A_{j \mu}^{a} & =g f^{a b c} A_{j \mu}^{b} c^{c}, & s b_{j}^{a} & =g f^{a b c} b_{j}^{b} c^{c}, \\
s \bar{c}_{i}^{a} & =g f^{a b c} \bar{c}_{i}^{b} c^{c}, & s c_{i}^{a} & =g f^{a b c} c_{i}^{b} c^{c},
\end{aligned}
$$

cancels the extra terms that arise in $\operatorname{det} \mathbb{A}_{j}^{1}$ and $\operatorname{det} \mathbb{C}_{i}^{1}$ when the gauge field $A_{\mu}^{a}$ is BRS-transformed. However, if $r=0$, the determinants $\operatorname{det} \mathbb{A}_{j}^{r}$ and $\operatorname{det} \mathbb{C}_{i}^{r}$ are not BRS invariant. Faddeev's and Slavnov's proposal [2,3] corresponds to taking $r=1$ and is the one we will be studying in this paper. For later convenience, though, we will keep $r$ explicit in our calculations.

The Feynman rules associated to the functional $Z_{r}[J, \chi, \eta, \bar{\eta}]$ are listed in Appendix A. Note that the determinant $\operatorname{det} \mathbb{A}_{j}^{r}$ has been defined in such a way that the vertices $A^{2 l+1}(l=3, \ldots, 6)$ have the same Feynman rule as $A_{j}^{2} A^{l}$. Note also that the Feynman rules for the vertices $b_{j} A A_{j}, \bar{c}_{i} A c_{i}$ and $\bar{c}_{i} A^{2} c_{i}$ depend explicitly on $r$. Keeping these two observations in mind, we move on to discussing how the Pauli-Villars determinants just introduced regularize the one-loop divergences generated by $S_{A}$. We start discussing first the case $r=0$. 


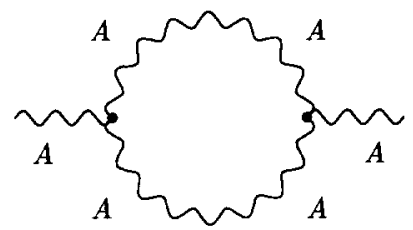

(a)

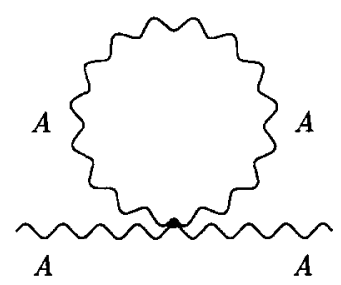

(c)

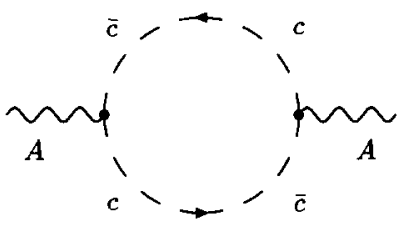

(e)

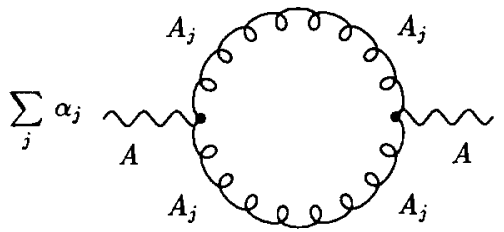

(b)

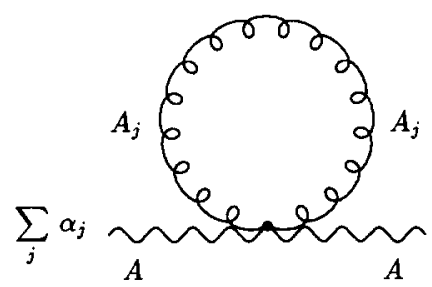

(d)

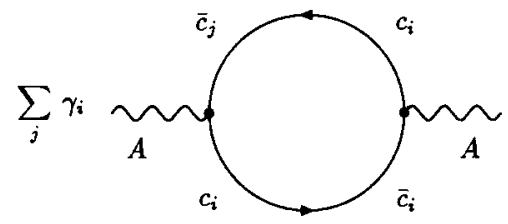

(f)

Fig. 1. One-loop regularization of the vacuum polarization tensor: diagrams that contribute for both $r=0,1$. Recall that the contribution of (f) depends on the value of $r$.

Gauge non-invariant case: $r=0$

We want to show that the vacuum polarization tensor and the vertices $\left\langle A^{3}\right\rangle_{1 P I}$ and $\left\langle A^{4}\right\rangle_{1 \text { PI }}$ at one loop generated by $Z_{0}[J, \chi, \eta, \bar{\eta}]$ in Eq. (2.12) are finite. Let us start with the vacuum polarization tensor. At one loop it receives contributions from the diagrams in Fig. 1. Let us look at the sum of the diagrams in Fig. 1a and 1b,

$$
\sigma_{1}=(1 \mathrm{a})+(1 \mathrm{~b}) .
$$

Recalling that $\alpha$-dependent contributions are superficially convergent, we have that in diagram (1a) only the $\alpha=0$ part contains divergences. Taking then $\alpha=0$, noting that by construction the vertices $A^{3}$ and $A^{2} A_{j}^{2}$ have the same Feynman rule, and using the identity 


$$
\frac{1}{q^{6}+q^{2} \Lambda^{4}+M_{j}^{2} \Lambda^{4}}=\frac{1}{q^{2}\left(q^{4}+\Lambda^{4}\right)}-\frac{M_{j}^{2} \Lambda^{4}}{q^{2}\left(q^{4}+\Lambda^{4}\right)\left(q^{6}+q^{2} \Lambda^{4}+M_{j}^{2} \Lambda^{4}\right)}
$$

for the propagator of the Pauli-Villars field $A_{j}$, it is trivial to see that the only superficially divergent integrals in $\sigma_{1}$ are quadratically divergent, do not depend on $M_{j}$ and pick an overall factor $1+\sum_{j} \alpha_{j}$. It thus follows that if we choose parameters $\alpha_{j}$ such that

$$
1+\sum_{j=1}^{J} \alpha_{j}=0
$$

the sum $\sigma_{1}$ becomes finite. In other words, diagram ( $\mathrm{lb}$ ) regularizes diagram (1a) if condition (2.17) is satisfied. A similar argument shows that the sum

$$
\sigma_{2}=(1 \mathrm{c})+(1 \mathrm{~d})
$$

is finite if again condition (2.17) is met. Let us finally consider the sum

$$
\sigma_{3}^{0}=(1 \mathrm{e})+(1 \mathrm{f}) \text {. }
$$

Noting that for $r=0$ the vertex $\bar{c}_{i} A c_{i}$ has the same Feynman rule as the vertex $\bar{c} A c$ and using the identity

$$
\frac{1}{q^{2}+m_{i}^{2}}=\frac{1}{q^{2}}-\frac{m_{i}^{2}}{q^{2}\left(q^{2}+m_{i}^{2}\right)}
$$

twice, we find that $\sigma_{3}^{0}$ contains quadratic and logarithmic divergences that do not depend on $m_{i}$ and that pick overall factors $1+\sum_{i} \gamma_{i}$, and $\sum_{i} \gamma_{i} m_{i}^{2}$, respectively. Hence imposing conditions

$$
\begin{aligned}
& 1+\sum_{i=1}^{I} \gamma_{i}=0, \\
& \sum_{i=1}^{I} \gamma_{i} m_{i}^{2}=0,
\end{aligned}
$$

we render the sum $(e)+(f)$ finite.

To summarize, if the parameters $\alpha_{j}$ and $\gamma_{i}$ and the masses $M_{j}$ and $m_{i}$ satisfy the Pauli-Villars conditions (2.17)-(2.21), diagrams (1b), (1d) and (1f) regularize diagrams (1a), (1c) and (1e) and the vacuum polarization tensor becomes finite. Analogous arguments show that one-loop divergences in the vertices $\left\langle A^{3}\right\rangle_{1 \mathrm{PI}}$ and $\left\langle A^{4}\right\rangle_{1 \mathrm{PI}}$ generated by $S_{A}$ are regularized if the very same conditions $(2.17)-(2.21)$ hold. This regularization procedure is very neat, but as already pointed out is not gauge invariant.

Gauge invariant case: $r=1$

We know that the Feynman rules are not the same for $r=1$ as for $r=0$. Hence there is no reason to assume that the regularization mechanism is the same in both cases. To 


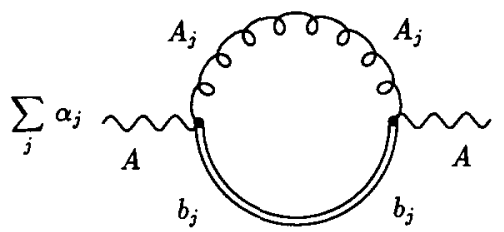

(a)

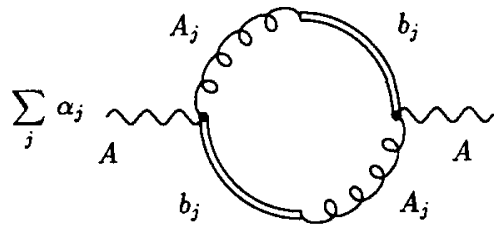

(b)

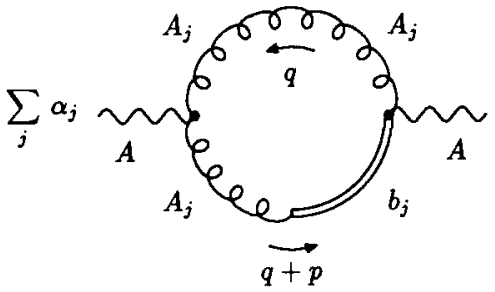

(c)

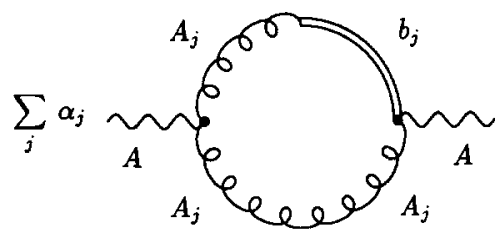

(d)

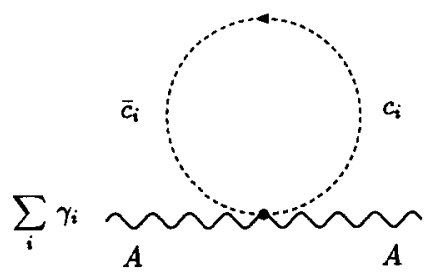

(e)

Fig. 2. One-loop regularization of the vacuum polarization tensor: diagrams that contribute only for $r=1$.

study regularization for $r=1$ in detail, let us consider again the vacuum polarization tensor. At one loop, it receives contributions from the diagrams in Fig. 1 and, very importantly, from those in Fig. 2. We first look at the diagrams in Fig. 1. Diagrams (1a) to (1d) are independent of $r$, so the same arguments as for $r=0$ imply that the sums $\sigma_{1}$ and $\sigma_{2}$ are finite provided condition (2.17) holds. As concerns the sum $\sigma_{3}^{0}$, we note that diagram (1e) is independent of $r$ but (1f) is not. Furthermore, the difference in between taking $r=1$ and $r=0$ in diagram (1f) is a superficially divergent integral. Therefore, after imposing conditions (2.20) and (2.21), we are left with unregularized divergences in $\sigma_{3}^{0}$. Next we look at the diagrams in Fig. 2, which we emphasize do not exist for $r=0$. Some trivial power counting shows that diagram (2a) is finite, while diagrams $(2 \mathrm{~b})$ to $(2 \mathrm{e})$ are superficially divergent. Hence, for the generating functional $Z_{1}[J, \chi, \eta, \bar{\eta}]$ to produce a finite vacuum polarization tensor, the divergences in (le) 
and (1f) must cancel the divergences in diagrams (2b) to (2e). In other words, the sum

$$
\sigma_{3}^{1}=(1 \mathrm{e})+(1 \mathrm{f})+(2 \mathrm{~b})+(2 \mathrm{c})+(2 \mathrm{~d})+(2 \mathrm{e})
$$

must be finite. All this makes obvious that regularization for $r=1$ does not go as for $r=0$. It also corrects some statements in the previous literature [3], where it is explicitly claimed that the terms that ensure manifest BRS invariance do not introduce new divergences into the game.

Let us find the conditions on $\alpha_{j}, \gamma_{i}, M_{j}$ and $m_{i}$ for which the sum $\sigma_{3}^{1}$ is finite. A look at the topology of the diagrams contributing to $\sigma_{3}^{1}$ reveals that an analysis of the type performed for $\sigma_{1}$ and $\sigma_{2}$ is not possible. Yet we have to seek for cancellations of divergences from different diagrams. To do this with rigour, we introduce a regulator $\mathcal{R}$, compute the diagrams at finite $\mathcal{R}$ and see for what values of $\alpha_{j}, \gamma_{i}, M_{j}$ and $m_{i}$ contributions divergent at $\mathcal{R}=0$ cancel upon summation. To isolate the divergences at $\mathcal{R}=0$ in each one of the diagrams, we proceed as follows. Consider for example diagrams (2c) and (2d). With the choice of momenta in Fig. 2 and after some Lorentz algebra $^{1}$, we have

$$
(2 \mathrm{c})+(2 \mathrm{~d})=r g^{2} c_{V} \sum_{j=1}^{J} \alpha_{j} \int_{\mathcal{R}} \frac{\mathrm{d}^{4} q}{(2 \pi)^{4}} \frac{N_{\mu \nu}}{(q+p)^{2} q^{2}\left(q^{6}+q^{2} \Lambda^{4}+M_{j}^{2} \Lambda^{4}\right)},
$$

where

$$
N_{\mu \nu}=2 q^{6}\left(q_{\mu} q_{\nu}-q^{2} g_{\mu \nu}\right)+O\left(q^{n}, 2 \leqslant n<6\right) .
$$

Terms in $N_{\mu \nu}$ of order five or less in $q^{\mu}$ give rise to integrals finite by power counting at $\mathcal{R}=0$. On the other hand, terms in $N_{\mu \nu}$ of order six or higher in $q^{\mu}$ give rise to superficially divergent integrals at $\mathcal{R}=0$. This means that as far as regularization is concerned it is enough to keep the terms written explicitly in Eq. (2.23). Using then the identities (2.16) and

$$
\frac{1}{q^{4}+\Lambda^{4}}=\frac{1}{q^{4}}-\frac{1}{q^{4}\left(q^{4}+\Lambda^{4}\right)}
$$

and retaining only integrals which are superficially divergent at $\mathcal{R}=0$, it is trivial to see that

$$
(2 \mathrm{c})+\left.(2 \mathrm{~d})\right|_{\mathrm{div}}=-r g^{2} c_{V} \delta^{a b} \sum_{j=1}^{J} \alpha_{j} \int_{\mathcal{R}} \frac{\mathrm{d}^{4} q}{(2 \pi)^{4}} \frac{2\left(g_{\mu \nu}-q_{\mu} q_{\nu}\right)}{(p+q)^{2}} .
$$

Proceeding in the same way for the other diagrams in $\sigma_{3}^{1}$, summing over diagrams and imposing condition (2.17), we obtain

\footnotetext{
${ }^{1}$ To perform the Lorentz algebra of the diagrams in this paper, we have used the algebraic package REDUCE $[10]$.
} 


$$
\begin{aligned}
& \left.\sigma_{3}^{1}(\mathcal{R})\right|_{\mathrm{div}}=-g^{2} c_{V} \delta^{a b}\left(1+\sum_{i=1}^{I} \gamma_{i}\right) \int_{\mathcal{R}} \frac{\mathrm{d}^{4} q}{(2 \pi)^{4}} \frac{4 q_{\mu} q_{\nu}+q_{\mu} p_{\nu}+p_{\mu} q_{\nu}}{q^{2}(q+p)^{2}} \\
& +2 g^{2} c_{V} \delta^{a b} \int_{\mathcal{R}} \frac{\mathrm{d}^{4} q}{(2 \pi)^{4}}\left\{\sum_{i=1}^{I} \gamma_{i}\left[\frac{g_{\mu \nu}}{q^{2}+m_{i}^{2}}+\frac{4 m_{i}^{2} q_{\mu} q_{\nu}}{q^{4}\left(q^{2}+m_{i}^{2}\right)}\right]+\frac{g_{\mu \nu}}{(q+p)^{2}}\right\} \\
& -g^{2} c_{V} \delta^{a b} \sum_{i=1}^{I} \gamma_{i} \int_{\mathcal{R}} \frac{\mathrm{d}^{4} q}{(2 \pi)^{4}} \frac{q_{\mu} p_{\nu}+p_{\mu} q_{\nu}+p_{\mu} p_{\nu}}{q^{2}(q+p)^{2}} .
\end{aligned}
$$

Assume we take as regulator $\mathcal{R}$ dimensional regularization, i.e. assume that

$$
\int_{\mathcal{R}} \frac{\mathrm{d}^{4} q}{(2 \pi)^{4}}=\frac{1}{\mu^{D-4}} \int \frac{\mathrm{d}^{D} q}{(2 \pi)^{D}}, \quad D=4+2 \varepsilon,
$$

with $\mu$ the dimensional regularization mass scale. Then, it is not difficult to see that

$$
\left.\sigma_{3}^{1}(\varepsilon)\right|_{\mathrm{div}}=\frac{g^{2} c_{V}}{16 \pi^{2}} \delta^{a b}\left[\left(1+\sum_{i=1}^{I} \gamma_{i}\right) \sigma_{\varepsilon}\left(p^{2} g_{\mu \nu}-p_{\mu} p_{\nu}\right)+\sum_{i=1}^{l} \gamma_{i} p^{2} g_{\mu \nu}\right]
$$

as $D \rightarrow 4$, where

$$
\sigma_{\varepsilon}=\frac{1}{3}\left[\frac{1}{\varepsilon}+\ln \left(\frac{p^{2}}{\mu^{2}}\right)+\gamma-\frac{8}{3}\right] \text {. }
$$

To get rid of the pole in Eq. (2.26) and end up with a finite $\sigma_{3}^{1}$, we choose the parameters $\gamma_{i}$ to satisfy the Pauli-Villars condition (2.20).

Putting everything together, we have that the regularized vacuum polarization tensor is finite for parameters $\alpha_{j}$ and $\gamma_{i}$ satisfying the Pauli-Villars conditions (2.17) and (2.20). Using the BRS identities and that the vacuum polarization tensor, the ghost self-energy and the $\bar{c} A c$-vertex are finite, it follows the three and four-point Green functions $\left\langle A^{3}\right\rangle_{1 \mathrm{PI}}$ and $\left\langle A^{4}\right\rangle_{1 P I}$ are also finite, provided of course conditions (2.17) and (2.20) hold. This completes the proof that the functional $Z_{1}[J, \chi, \eta, \bar{\eta}]$ generates finite one-loop Green functions. There are two important comments to be made at this point:

Comment 1. It is clear that regularization does not require condition (2.21), contrarily to what is claimed in Ref. [3] and all papers thereafter.

Comment 2. Divergences from isolated Feynman diagrams in $\sigma_{3}^{1}$ get regularized only after integration at finite $\mathcal{R}$. This is in contrast with what happens for $\sigma_{1}$ and $\sigma_{2}$, where regularization takes place algebraically prior to integration. Here we have taken dimensional regularization as regulator $\mathcal{R}$ because it preserves BRS invariance. Assume that instead we take a cut-off $Q$ such that $|p|, m_{i} \ll Q$. Some elementary integration in Eq. (2.25) then leads to

$$
\left.\sigma_{3}^{l}(Q)\right|_{\mathrm{div}}=\frac{g^{2} c_{V}}{16 \pi^{2}} \delta^{a b}\left[\left(1+\sum_{i=1}^{I} \gamma_{i}\right)\left(Q^{2} g_{\mu \nu}+\sigma_{Q}^{\prime} p^{2} g_{\mu \nu}-\sigma_{Q}^{\prime \prime} p_{\mu} p_{\nu}\right)\right.
$$




$$
\left.+\sum_{i=1}^{l} \gamma_{i}\left(p^{2} g_{\mu \nu}-\frac{1}{2} p_{\mu} p_{\nu}\right)\right]
$$

where

$$
\sigma_{Q}^{\prime}=\frac{1}{3} \ln \left(\frac{Q^{2}}{p^{2}}\right)+\frac{11}{18}, \quad \sigma_{Q}^{\prime \prime}=\frac{1}{3} \ln \left(\frac{Q^{2}}{p^{2}}\right)-\frac{1}{18} .
$$

Again we see that for $\gamma_{i}$ satisfying Eq. (2.20), the sum $\sigma_{3}^{1}$ is finite. Comparing Eq. (2.26) with Eq. (2.27), we note that the finite part that is left in $\left.\sigma_{3}^{1}\right|_{\text {div }}$ after imposing condition (2.20) is however different. This implies that different $\mathcal{R}^{\prime}$ s give different local contributions to $\sigma_{3}^{1}$. As regards the non-local part of $\sigma_{3}^{1}$, it is the same for all $\mathcal{R}$ since it arises only from integrals which are finite by power counting at $\mathcal{R}=0$.

To sum up, Pauli-Villars determinants with $r=1$ are BRS invariant, but checking that they provide the necessary regularization requires introducing a pre-regulator $\mathcal{R}$. This means strictly speaking that $r=1$ Pauli-Villars determinants do not provide an acceptable regularization. Still one could argue that since non-local contributions are independent of the choice of pre-regulator $\mathcal{R}, r=1$ Pauli-Villars regularization is good enough. In Sections 4 and 5 we show that this is not either the case, since the determinants $\operatorname{det} \mathbb{A}_{j}^{1}$ and $\operatorname{det} \mathbb{C}_{i}^{1}$ introduce unphysical logarithmic radiative corrections.

\section{The renormalized theory}

So far we have a BRS invariant regularization prescription that involves the masses $\Lambda, M_{j}$ and $m_{i}$ (and a pre-regulator $\mathcal{R}$ which from now on we take it to be dimensional regularization). The next step is to define the renormalized theory. There are several requirements that the resulting renormalized theory must satisfy for it to make sense. One of them is that the renormalized Green functions satisfy the BRS identities. To date, there is no agreement in the literature [8] as whether starting from the generating functional $Z_{1}[J, \chi, \eta, \bar{\eta}]$ it is possible to define renormalized Green functions compatible with BRS invariance. In this section we show that this is indeed possible at one loop at least.

To study the BRS identities, it is convenient to work with the effective action. The effective action associated to the functional $Z_{1}[J, \chi, \eta, \bar{\eta}]$ is defined in two steps. One first introduces external fields $K^{a \mu}$ and $H^{a}$ coupled respectively to the non-linear BRS transforms $s A_{\mu}^{a}$ and $s c^{a}$ so that the generating functional becomes

$$
\begin{aligned}
Z_{1}[J, \chi, \eta, \bar{\eta} ; K, H]= & \int \mathcal{D} A \mathcal{D} b \mathcal{D} \bar{c} \mathcal{D} c \mathrm{e}^{-\left(S_{A}+S_{J}+S_{\text {ext }}\right)} \\
& \times \prod_{j=1}^{J}\left(\operatorname{det} \mathbb{A}_{j}^{l}\right)^{-\alpha_{j} / 2} \prod_{i=1}^{I}\left(\operatorname{det} \mathbb{C}_{i}^{1}\right)^{\gamma_{i}},
\end{aligned}
$$

with $S_{\text {ext }}$ given by 


$$
S_{\mathrm{ext}}=\int \mathrm{d}^{4} x\left[K^{a \mu}\left(s A_{\mu}^{a}\right)+H^{a}\left(s c^{a}\right)\right] .
$$

And secondly, one writes

$$
Z_{1}[J, \chi, \eta, \bar{\eta} ; K, H]=\exp (-W[J, \chi, \eta, \bar{\eta} ; K, H])
$$

and performs a Legendre transformation on $W[J, \chi, \eta, \bar{\eta} ; K, H]$ and on the sources $J^{a \mu}$, $\chi^{a}, \eta^{a}$ and $\bar{\eta}^{a}$. The Legendre transform of $W[J, \chi, \eta, \bar{\eta} ; K, H]$ is the regularized effective action, a functional of mass dimension four and ghost number zero that depends on the Legendre fields $A_{\mu}^{a}, b^{a}, \bar{c}^{a}, c^{a}$ and on the external fields $K^{a \mu}, H^{a}$ and that generates 1PI Green functions. In what follows we will denote by the letter $\psi$ the set of fields $\{\psi\}=$ $\left\{A_{\mu}^{a}, b^{a}, \bar{c}^{a}, c^{a}, K^{a \mu}, H^{a}\right\}$ and write $\Gamma_{A M_{j} m_{i}}[\psi]$ for the regularized effective action, our conventions for the mass dimensions $d_{\psi}$ and the ghost numbers $\mathrm{gh}_{\psi}$ of the fields being $\left\{d_{\psi}\right\}=\{1,2,1,1,2,2\}$ and $\left\{\mathrm{gh}_{\psi}\right\}=\{0,0,-1,1,-1,-2\}$. It is very easy to see that all 1PI Feynman diagrams with external fields $K^{a \mu}, H^{a}$ are superficially convergent. This ensures that the manipulations performed above to arrive at the effective action $\Gamma_{\Lambda M_{j} m_{i}}[\psi]$ do not introduce divergences.

We will write

$$
\Gamma_{A M_{j} m_{i}}[\psi]=\Gamma_{A}^{(0)}[\psi]+\Gamma_{A M_{j} m_{i}}^{(1)}[\psi]+O\left(\hbar^{2}\right),
$$

where by definition the tree-level contribution $\Gamma_{A}^{(0)}[\psi]$ is given by

$$
\Gamma_{A}^{(0)}[\psi]=S_{A}+S_{\text {ext }}
$$

and $\Gamma_{A M_{j} m_{i}}^{(1)}[\psi]$ collects all one-loop corrections. Since the action $S_{A}$ and the determinants $\operatorname{det} \mathbb{A}_{j}^{l}$ and $\operatorname{det} \mathbb{C}_{i}^{l}$ are BRS invariant, $\Gamma_{\lambda M_{j} m_{i}}[\psi]$ satisfies the BRS identities. At tree level and one loop, they take the form

$$
\begin{aligned}
\Delta_{A} \Gamma_{A}^{(0)}[\psi] & =0 \\
\Delta_{A} \Gamma_{\Lambda M_{j} m_{i}}^{(1)}[\psi] & =0,
\end{aligned}
$$

where $\Delta_{A}$ is the Slavnov-Taylor operator for the action $\Gamma_{A}^{(0)}[\psi]$. The operator $\Delta_{A}$ can be written as

$$
\Delta_{\Lambda}=\Delta+\int \mathrm{d}^{4} x \frac{\delta S_{2}}{\delta A_{\mu}^{a}} \frac{\delta}{\delta K^{a \mu}},
$$

where

$$
\Delta=\int \mathrm{d}^{4} x\left[\frac{\delta \Gamma^{(0)}}{\delta A_{\mu}^{a}} \frac{\delta}{\delta K^{a \mu}}+\frac{\delta \Gamma^{(0)}}{\delta K^{a \mu}} \frac{\delta}{\delta A_{\mu}^{a}}+\frac{\delta \Gamma^{(0)}}{\delta c^{a}} \frac{\delta}{\delta H^{a}}+\frac{\delta \Gamma^{(0)}}{\delta H^{a}} \frac{\delta}{\delta c^{a}}+b^{a} \frac{\delta \Gamma^{(0)}}{\delta \bar{c}^{a}}\right]
$$

is the Slavnov-Taylor operator for Yang-Mills theory and $S_{2}$ is as in Eq. (2.7), the action $\Gamma^{(0)}[\psi]$ being the tree-level Yang-Mills action

$$
\Gamma^{(0)}[\psi]=S_{\mathrm{YM}}+S_{\mathrm{GF}}+S_{\mathrm{ext}} .
$$


For later use we recall that $\Delta$ is nilpotent, $\Delta^{2}=0$, and satisfies $\Delta S_{Y M}=0$. Eq. (3.2) is trivially satisfied and does not provide any new information. We want to study the structure of the 1PI Green functions that the regularized effective action $\Gamma_{A M_{j} m_{i}}[\psi]$ generates and the constraints that Eq. (3.3) imposes on them.

Let us then consider the regularized vacuum polarization tensor $\Pi_{\mu \nu}^{a b}\left(p, \Lambda, M_{j}, m_{i}\right)$. Manifest BRS invariance implies that up to one loop it will be of the form

$$
\Pi_{\mu \nu}^{a b}\left(p, \Lambda, M_{j}, m_{i}\right)=\left[1+\frac{g^{2} c_{V}}{16 \pi^{2}} \Pi\left(p, \Lambda, M_{j}, m_{i}\right)\right] \delta^{a b}\left(p^{2} g_{\mu \nu}-p_{\mu} p_{\nu}\right),
$$

where $\Pi\left(p, A, M_{j}, m_{i}\right)$ is a dimensionless function of its arguments. As $\Pi\left(p, A, M_{j}, m_{i}\right)$ is dimensionless, it can be written without loss of generality as a function of $p^{2} / A^{2}$, $p^{2} / M_{j}^{2}$ and $p^{2} / m_{i}^{2}$. Power counting implies that in the limit $\Lambda, M_{j}, m_{i} \rightarrow \infty$

$$
\Pi\left(p, \Lambda, M_{j}, m_{i}\right)=A_{2} \ln \left(\frac{p^{2}}{\Lambda^{2}}\right)+B_{2} \sum_{j=1}^{J} \alpha_{j} \ln \left(\frac{p^{2}}{M_{j}^{2}}\right)+C_{2} \sum_{i=1}^{I} \gamma_{i} \ln \left(\frac{p^{2}}{m_{i}^{2}}\right)+\pi_{0},
$$

with $A_{2}, B_{2}, C_{2}$ and $\pi_{0}$ coefficients that depend on $\alpha$. Assume more generally now that $G^{(0)}\left(p_{e}\right)$ denotes an arbitrary 1PI Yang-Mills Green function at tree level with $\left\{p_{e}\right\}=\left\{p_{1}^{\mu}, \ldots, p_{E}^{\mu}\right\}$ independent external momenta. Manifest BRS invariance and power counting imply that in the limit $\Lambda, M_{j}, m_{i} \rightarrow \infty$ the corresponding regularized Green function, $G\left(p_{e}, A, M_{j}, m_{i}\right)$, can be written up to one loop without loss of generality as

$$
G\left(p_{e}, \Lambda, M_{j}, m_{i}\right)=G^{(0)}\left(p_{e}\right)+\frac{g^{2} c_{V}}{16 \pi^{2}}\left[\phi_{G}\left(p, \Lambda, M_{j}, m_{i}\right) G^{(0)}\left(p_{e}\right)+G^{\mathrm{fin}}\left(p_{e}\right)\right],
$$

where $\phi_{G}\left(p, \Lambda, M_{j}, m_{i}\right)$ is given by

$$
\phi_{G}\left(p, \Lambda, M_{j}, m_{i}\right)=A_{G} \ln \left(\frac{p^{2}}{\Lambda^{2}}\right)+B_{G} \sum_{j=1}^{J} \alpha_{j} \ln \left(\frac{p^{2}}{M_{j}^{2}}\right)+C_{G} \sum_{i=1}^{l} \gamma_{i} \ln \left(\frac{p^{2}}{m_{i}^{2}}\right)
$$

and $G^{\text {fin }}\left(p_{e}\right)$ collects all finite contributions. The momentum $p^{\mu}$ in Eq. (3.8) is a nonzero linear combination of the external momenta and can be chosen arbitrarily since

$$
\ln \left(p^{2} / \Lambda^{2}\right)=\ln \left(p^{2} / \Lambda^{2}\right)+\ln \left(p^{2} / p^{2}\right)
$$

for all $p^{2} \neq 0$. Obviously, different choices of $p^{\mu}$ give different finite parts $G_{\text {fin }}\left(p_{e}\right)$ but the same coefficients $A_{G}, B_{G}$ and $C_{G}$. Introducing a renormalization mass scale $\kappa$ and using the Pauli-Villars conditions (2.17) and (2.20), we can cast $G\left(p_{e}, \Lambda, M_{j}, m_{i}\right)$ as

$$
G\left(p_{e}, \Lambda, M_{j}, m_{i}\right)=G^{(0)}\left(p_{e}\right)+\frac{g^{2} c_{V}}{16 \pi^{2}}\left[\phi_{G}\left(\kappa, \Lambda, M_{j}, m_{i}\right) G^{(0)}\left(p_{e}\right)+G_{\kappa}^{\mathrm{fin}}\left(p_{e}\right)\right],
$$


where now

$$
\phi_{G}\left(\kappa, \Lambda, M_{j}, m_{i}\right)=A_{G} \ln \left(\frac{\kappa^{2}}{\Lambda^{2}}\right)+B_{G} \sum_{j=1}^{J} \alpha_{j} \ln \left(\frac{\kappa^{2}}{M_{j}^{2}}\right)+C_{G} \sum_{i=1}^{l} \gamma_{i} \ln \left(\frac{\kappa^{2}}{m_{i}^{2}}\right)
$$

and

$$
G_{\kappa}^{\mathrm{fin}}\left(p_{e}\right)=\left(A_{G}-B_{G}-C_{G}\right) \ln \left(\frac{p^{2}}{\kappa^{2}}\right) G^{(0)}\left(p_{e}\right)+G^{\mathrm{fin}}\left(p_{e}\right) .
$$

From now on we will be using the following notation. Subindices $G=2,3,4$ will refer to the two-, three- and four-point Green functions $\left\langle A^{2}\right\rangle_{1 \mathrm{PI}},\left\langle A^{3}\right\rangle_{1 \mathrm{PI}}$ and $\left\langle\mathrm{A}^{4}\right\rangle_{1 \mathrm{PI}}$, respectively; subindices $G=2$ and a tilde and $G=3$ and a tilde will refer to $\langle\bar{c} c\rangle_{1 \mathrm{PI}}$ and $\langle\bar{c} A c\rangle_{1 \mathrm{PI}}$; and subindices $G=K$ and $G=H$ will refer to $\langle K A c\rangle_{1 \mathrm{PI}}$ and $\langle H c c\rangle_{1 \mathrm{PI}}$. Note that since the Pauli-Villars fields do not couple to the Faddeev-Popov ghosts nor to the external fields, we have

$$
\tilde{B}_{2}=\tilde{B}_{3}=0, \quad \tilde{C}_{2}=\tilde{C}_{3}=0, \quad B_{K}=C_{K}=0, \quad B_{H}=C_{H}=0 .
$$

To find the constraints on the coefficients $A_{G}, B_{G}$ and $C_{G}$ that the BRS identity (3.3) imposes, we note that one-loop 1PI diagrams with $K^{a \mu}$ external legs have $B_{K}=C_{K}=0$. Hence these diagrams give contributions to $\Gamma_{A M_{j} m_{i}}^{(1)}[\psi]$ that when $\Lambda, M_{j}, m_{i} \rightarrow \infty$ are either finite or diverge as $\ln \left(\kappa^{2} / \Lambda^{2}\right)$. Recalling that $S_{2}$ has a overall factor $1 / \Lambda^{4}$, this implies that

$$
\lim _{\lambda, M_{j}, m_{i} \rightarrow \infty} \int \mathrm{d}^{4} x \frac{\delta S_{2}}{\delta A_{\mu}^{a}} \frac{\delta}{\delta K^{a \mu}} \Gamma_{\lambda M_{j} m_{i}}^{(1)}[\psi]=0 .
$$

From our considerations above it follows that in the limit $\Lambda, M_{j}, m_{i} \rightarrow \infty$ the one-loop contribution to the regularized effective action takes the form

$$
\Gamma_{A M_{j} m_{i}}^{(1)}[\psi]=\Gamma_{A M_{j} m_{i} \kappa}^{(1) \operatorname{div}}[\psi]+\Gamma_{\kappa}^{(1) \text { fin }}[\psi]+\text { v.t. },
$$

where $\Gamma_{A M_{j} m_{i} \kappa}^{(1) \text { div }}[\psi]$ generates the $\phi_{G}$-contributions in Eq. $(3.10), \Gamma_{\kappa}^{(1) \text { fin }}[\psi]$ generates finite one-loop radiative corrections and "v.t." collects contributions that vanish as $\Lambda, M_{j}, m_{i} \rightarrow \infty$. Eqs. (3.13) and (3.14) imply that the BRS identity (3.3) reduces in the limit $A, M_{j}, m_{i} \rightarrow \infty$ to

$$
\begin{gathered}
\Delta \Gamma_{\Delta M_{j} m_{i} \kappa}^{(1) \operatorname{div}}[\psi]=0, \\
\Delta \Gamma_{\kappa}^{(1) \text { fin }}[\psi]=0 .
\end{gathered}
$$

Using that by definition $\Gamma_{A M_{j} m_{i} \kappa}^{(1) \text { div }}[\psi]$ generates the $\phi_{G}$-contributions to the Green functions in Eq. (3.10), it is not difficult to see after some algebra that Eq. (3.15) implies that the coefficients $A_{G}, B_{G}$ and $C_{G}$ satisfy

$$
A_{2}-A_{3}=A_{3}-A_{4}=\tilde{A_{2}}-\tilde{A_{3}} \text {, }
$$




$$
\begin{aligned}
& B_{2}=B_{3}=B_{4}, \\
& C_{2}=C_{3}=C_{4} .
\end{aligned}
$$

Once we know the form of the divergences in the regularized Green functions when the regulators are sent to infinity, we are in a position to renormalize the theory.

To define a one-loop renormalized Yang-Mills theory consistent with gauge invariance, we have to provide a one-loop renormalized effective action

$$
\Gamma_{R}[\psi]=\Gamma^{(0)}[\psi]+\Gamma_{R}^{(1)}[\psi]+O\left(\hbar^{2}\right)
$$

that generates finite 1PI Green functions and that satisfies the Yang-Mills BRS identities. We recall that the latter take the form up to one loop

$$
\begin{aligned}
& \Delta \Gamma^{(0)}[\psi]=0, \\
& \Delta \Gamma_{R}^{(1)}[\psi]=0 .
\end{aligned}
$$

For $\Gamma_{R}^{(1)}[\psi]$ we take

$$
\Gamma_{R}^{(1)}[\psi]=\Gamma_{\kappa}^{(1) \text { fin }}[\psi]+c_{1} S_{\mathrm{YM}}+c_{2} \Delta X[\psi],
$$

where $\Gamma_{\kappa}^{(1) \text { fin }}[\psi]$ is the same as in Eq. (3.14), $c_{1}$ and $c_{2}$ are arbitrary coefficients of order $\hbar$ and $X[\psi]$ is the most general local integrated functional of mass dimension 3 and ghost number -1 that can be constructed with the fields $\psi$. The coefficients $c_{1}$ and $c_{2}$ and the functional $X$ are taken to be independent of the scale $\kappa$. By construction, $\Gamma_{R}[\psi]$ generates finite Green functions since nothing in it depends on the cut-offs $A, M_{j}$ and $m_{i}$. As regards the BRS identities, Eq. (3.21) is trivially satisfied, so we only have to show that Eq. (3.22) holds. That this is the case follows from Eq. (3.3) and the properties $\Delta S_{\mathrm{YM}}=0$ and $\Delta^{2}=0$. Hence the effective action $\Gamma_{R}[\psi]$ with $\Gamma_{R}^{(1)}[\psi]$ as in Eq. (3.23) defines the most general one-loop renormalized theory compatible with BRS invariance.

Note that we have defined the renormalized theory via an effective action without making reference to any bare theory. This way to proceed can be viewed as a Bogoliubov subtraction $\mathbf{T}$ on the regularized effective action, namely

$$
\Gamma_{R}[\psi]=\lim _{A, M_{j}, m_{i} \rightarrow \infty}(1-\mathbf{T}) \Gamma_{A M_{j} m_{i}}[\psi],
$$

where

$$
\mathbf{T} \Gamma_{A M_{j} m_{i}}[\psi]=\Gamma_{\lambda m_{j} m_{i} \kappa}^{(1) \operatorname{div}}[\psi]-c_{1} S_{\mathrm{YM}}-c_{2} \Delta X[\psi] .
$$

The first term on the right-hand side in Eq. (3.25) removes the divergences in the regularized effective action when the regulators are sent to infinity. After subtracting this term and taking the limit $\Lambda, M_{j}, m_{i} \rightarrow \infty$, we are left with $\Gamma_{\kappa}^{(1)}$ fin $[\psi]$. The second and third terms account for arbitrary finite BRS-invariant local renormalizations. This follows from the fact [11] that the most general solution to the equation $\Delta Y[\psi]=0$ over the space of local integrated functionals of mass dimension 4 and ghost number zero is precisely of the form $c_{1} S_{\mathrm{YM}}+c_{2} \Delta X[\psi]$. All this implies that the subtraction 
T above gives the most general BRS invariant renormalized effective action that can be defined taking as starting point the regularized effective action provided by the regularization prescription we are considering ${ }^{2}$. We would like to finish this section with two comments.

Comment 1. The regularized effective action $\Gamma_{A M_{j} m_{i}}[\psi]$ from which the renormalized effective action $\Gamma_{R}[\psi]$ has been defined is consistent with a quantum action principle [11]. Thus the 1PI Green functions generated by $\Gamma_{R}[\psi]$ satisfy the renormalization group equations.

Comment 2. From the point of view of Yang-Mills theory, the masses $A, M_{j}$ and $m_{i}$ are regulators on the same footing and hence there is no reason why subtraction of divergences should be done in steps. What the $\mathbf{T}$ subtraction in Eqs. (3.24), (3.25) does is precisely to subtract the divergences associated to $\Lambda, M_{j}$ and $m_{i}$ at the same time. If, say, one performs a first renormalization to subtract the divergences that occur at finite $\Lambda$ when $M_{j}$ and $m_{i}$ are sent to infinity and then performs a second renormalization to subtract the divergences that arise when $\Lambda$ goes to infinity, one finds the same inconsistencies as in Ref. [8].

\section{The wrong beta function, a no-go result}

In this section we compute the beta function and the anomalous dimensions of the fields. To do this, we use the renormalization group equation.

\subsection{The renormalization group equations}

Let us recall that if $G_{R}\left(p_{e}, \kappa, g, \alpha\right)$ denotes a renormalized 1PI Yang-Mills Green function with $N_{\psi}$ external legs of type $\psi$, the renormalization group equation takes the form

$$
\left[\kappa \frac{\partial}{\partial \kappa}+\beta(g) \frac{\partial}{\partial g}+\frac{1}{2} \sum_{\psi} \gamma_{\psi}(g) N_{\psi}+\delta(g) \frac{\partial}{\partial \alpha}\right] G_{R}\left(p_{e}, \kappa, g, \alpha\right)=0,
$$

where the coefficients $\beta(g), \gamma_{\psi}(g)$ and $\delta(g)$ are power series in $g$,

$$
\begin{aligned}
\beta(g) & =\beta_{1} g+\beta_{2} g^{2}+\ldots, \\
\gamma_{\psi}(g) & =\gamma_{\psi, 1} g+\gamma_{\psi, 2} g^{2}+\ldots, \\
\delta(g) & =\delta_{1} g+\delta_{2} g^{2}+\ldots
\end{aligned}
$$

The renormalization group equation is an equation in the coefficients $\beta(g), \gamma_{\psi}(g)$ and $\delta(g)$ and holds for all values of the external momenta $p_{e}^{\mu}$ and the coupling constant $g$. We want to solve this equation up to one loop using as data the renormalized Green functions generated by the effective action $\Gamma_{R}[\psi]$ defined in the previous section.

\footnotetext{
${ }^{2}$ For further details on Bogoliubov-type subtractions for regularization methods containing higher covariant derivatives see Ref. [12].
} 
So let us consider the vacuum polarization tensor generated by $\Gamma_{R}[\psi]$. We remind ourselves that it has the form

$$
\begin{aligned}
\Pi_{R}^{a b \nu}(p, \kappa, g, \alpha)= & -\left\{1+\frac{g^{2} c_{V}}{16 \pi^{2}}\left[z_{2}(\alpha) \ln \left(\frac{p^{2}}{\kappa^{2}}\right)+\pi_{0}(\alpha)\right]\right\} \delta^{a b}\left(p^{2} g_{\mu \nu}-p_{\mu} p_{\nu}\right) \\
& +O\left(g^{4}\right),
\end{aligned}
$$

where for convenience we have introduced the notation

$$
z_{2}(\alpha)=A_{2}-B_{2}-C_{2}
$$

and $\pi_{0}(\alpha)$ is a constant that depends on $\alpha$ and that already includes local finite contributions from the terms $c_{1} S_{Y M}$ and $c_{2} \Delta X$ in Eq. (3.23). Substituting $G_{R}\left(p_{e}, \kappa, g, \alpha\right)$ in Eq. (4.1) by $\Pi_{R}^{a b}(p, \kappa, g, \alpha)$, we obtain for the left-hand side a power series in $g$. For the equation to hold for all $g$, the coefficients of this series must vanish independently. This gives to orders $g, g^{2}$ and $g^{3}$ the following three equations:

$$
\begin{aligned}
\gamma_{A, 1}=0, & \\
\gamma_{A, 2}+\frac{c_{V}}{16 \pi^{2}} & {\left[2 \beta_{1} \pi_{0}(\alpha)-2 z_{2}(\alpha)+\beta_{1} \ln \left(\frac{p^{2}}{\kappa^{2}}\right)\right]=0, } \\
\gamma_{A, 3}+\frac{c_{V}}{16 \pi^{2}} & \left\{2 \beta_{2} \pi_{0}(\alpha)+\gamma_{A, 1}+\delta_{1} \frac{\partial \pi_{0}(\alpha)}{\partial \alpha}\right. \\
+ & {\left.\left[\left(2 \beta_{2}+\gamma_{A, 1}\right) z_{2}(\alpha)+\delta_{1} \frac{\partial z_{2}(\alpha)}{\partial \alpha}\right] \ln \left(\frac{p^{2}}{\kappa^{2}}\right)\right\}=0 . }
\end{aligned}
$$

Since the renormalization group equation holds for all values of $p^{\mu}$, terms independent of $p^{\mu}$ and terms proportional to $\ln \left(p^{2} / \kappa^{2}\right)$ must vanish separately. This implies in particular

$$
\begin{aligned}
\gamma_{A, 1} & =0, & \gamma_{A, 2} & =\frac{c_{V}}{8 \pi^{2}} z_{2}(\alpha), \\
\beta_{1} & =0, & \beta_{2} & =\frac{1}{2 z_{2}(\alpha)} \frac{\partial z_{2}(\alpha)}{\partial \alpha} \delta_{1} .
\end{aligned}
$$

Let us next take the renormalized ghost self-energy. Our arguments in the previous section implies that it has the form

$$
\Omega_{R}^{a b}(p, \kappa, g, \alpha)=\left\{1+\frac{g^{2} c_{V}}{16 \pi^{2}}\left[\tilde{z}_{2}(\alpha) \ln \left(\frac{p^{2}}{\kappa^{2}}\right)+\omega_{0}(\alpha)\right]\right\} \delta^{a b} p^{2}+O\left(g^{4}\right),
$$

where $\tilde{z_{2}}(\alpha)$ stands for

$$
\tilde{z}_{2}(\alpha)=\tilde{A}_{2}
$$

and $\omega(\alpha)$ is an $\alpha$-dependent constant. Proceeding in the same way as for the vacuum polarization tensor, it is straightforward to see that the renormalization group equation for the ghost self-energy yields 


$$
\begin{aligned}
\gamma_{c, 1}+\gamma_{\bar{c}, 1} & =0, & \gamma_{c, 2}+\gamma_{\bar{c}, 2} & =\frac{c_{V}}{4 \pi^{2}} \tilde{z}_{2}(\alpha), \\
\beta_{1} & =0, & \beta_{2} & =\frac{1}{2 \tilde{z}_{2}(\alpha)} \frac{\partial \tilde{z}_{2}(\alpha)}{\partial \alpha} \delta_{1} .
\end{aligned}
$$

Let us finally look at the renormalization group equation for the $\bar{c} A c$-vertex. If $k^{\mu}$ and $p^{\mu}$ denote the momenta of the incoming ghost and the outgoing antighost, the vertex can be written without loss of generality as

$$
\begin{aligned}
V_{R}^{a b c}(p, k, \kappa, g, \alpha)=-i g f^{a b c}\{ & {\left[1+\frac{g^{2} c_{V}}{16 \pi^{2}} \tilde{z}_{3}(\alpha) \ln \left(\frac{p^{2}}{\kappa^{2}}\right)\right] p_{\mu} } \\
& \left.+\frac{g^{2} c_{V}}{16 \pi^{2}} V_{\mu}^{\mathrm{fin}}(k, p, \alpha)\right\}+O\left(g^{5}\right),
\end{aligned}
$$

where the coefficient $\tilde{z_{3}}(\alpha)$ is given by

$$
\tilde{z}_{3}(\alpha)=\tilde{A}_{3}
$$

and $V_{\mu}^{\text {fin }}(k, p, \alpha)$ contains local as well as non-local finite $\kappa$-independent radiative corrections. Using Eq. (4.6) as input in the renormalization group equation, Eq. (4.1), expanding in powers of $g$ up to order two and using our previous results for $\beta_{1}$ and the anomalous dimensions $\gamma_{\psi, 1}$ and $\gamma_{\psi, 2}$, it is very easy to see that

$$
\beta_{2}=0, \quad \beta_{3}=\frac{c_{V}}{16 \pi^{2}}\left[2 \tilde{z}_{3}(\alpha)-2 \tilde{z}_{2}(\alpha)-z_{2}(\alpha)\right] .
$$

The first one of these equation implies in turn that

$$
\delta_{1}=0 \text {. }
$$

Thus the renormalization group coefficients to lowest order can all be expressed in terms of $z_{2}(\alpha), \tilde{z}_{2}(\alpha)$ and $\tilde{z}_{3}(\alpha)$. To actually compute $z_{2}(\alpha), \tilde{z}_{2}(\alpha)$ and $\tilde{z}_{3}(\alpha)$, we must explicitly compute the limit $\Lambda, M_{j}, m_{i} \rightarrow \infty$ of the regularized vacuum polarization tensor, the regularized ghost self-energy and the regularized $\bar{c} A c$-vertex. We do this in the sequel.

\subsection{Explicit computations}

We begin with the vacuum polarization tensor $\Pi_{\mu \nu}^{a b}\left(p, \Lambda, M_{j}, m_{i}\right)$. At one loop, it receives contributions from the Feynman diagrams in Figs. 1 and 2 and in the limit $\Lambda, M_{j}, m_{i} \rightarrow \infty$ it takes the form in Eqs. (3.6) and (3.7). A look at the Feynman rules in Appendix A shows that

(i) diagrams (1e) and (2b) do not depend on any mass at all,

(ii) diagrams ( la) and (1c) depend on $A$,

(iii) diagrams (1f) and (2e) depend on $m_{i}$, and

(iv) diagrams (1b), (1d), (2a), (2c) and (2d) depend on both $\Lambda$ and $M_{j}$.

This implies that the coefficient $C_{2}$ in Eq. (3.7) is unambiguous, whereas the coefficients $A_{2}$ and $B_{2}$ depend on the path in $\left(\Lambda, M_{j}\right)$-space followed to approach $\Lambda, M_{j}=\infty$. Our calculations in Appendix B yield for $C_{2}$ the value 


$$
C_{2}=-\frac{1}{3}
$$

As regards $A_{2}$ and $B_{2}$, we consider in this paper paths of type

$$
\Lambda, M_{j} \rightarrow \infty \quad \text { with } \quad \frac{M_{j}}{\Lambda}=\theta_{j} \quad \text { and } \quad 0 \leqslant \theta_{j} \leqslant \infty .
$$

The path $\theta_{j}=\infty$ corresponds to first taking the limit $M_{j} \rightarrow \infty$ at finite $A$, and then taking the limit $A \rightarrow \infty$. The path $\theta_{j}=0$ on the contrary corresponds to first taking the limit $A \rightarrow \infty$ at finite $M_{j}$, and then taking the limit $M_{j} \rightarrow \infty$. The calculations in Appendix B show that

$$
\begin{aligned}
& \theta_{j}=\infty: \quad A_{2}=6-\frac{\alpha}{2} \quad B_{2}=4 \\
& \theta_{j}=0: A_{2}=-\frac{3+\alpha}{2} \quad B_{2}=-\frac{21}{6} \\
& 0<\theta_{j}<\infty: \quad A_{2}=2-\frac{\alpha}{2} \quad B_{2}=0 .
\end{aligned}
$$

We see that even though $A_{2}$ and $B_{2}$ are different for different $\theta_{j}$, the combination $A_{2}-B_{2}$ is the same. These results yield for $z_{2}(\alpha)$ in Eq. (4.3)

$$
z_{2}(\alpha)=\frac{7}{3}-\frac{\alpha}{2}
$$

It is important to note that in general the coefficients $A_{G}, B_{G}$ and $C_{G}$ are not important by themselves. What is really important are the combinations $z_{G}(\alpha)=A_{G}-B_{G}-C_{G}$, since they carry the $\kappa$-dependence of the renormalized Green functions. Note as a matter of fact that the beta function depends precisely on these combinations. In a sense, we can think of every $\left(\Lambda, M_{j}\right)$-path, equivalently of every set $\left\{A_{G}, B_{G}, C_{G}\right\}$, as defining a different regularization method for which the BRS identities (3.17)-(3.19) hold. Since we will extensively refer later on to the renormalized vacuum polarization tensor, let us put Eqs. (4.2) and (4.13) together:

$$
\begin{aligned}
\Pi_{R \mu \nu}^{a b}(p, \kappa, g, \alpha)= & -\left\{1+\frac{g^{2} c_{V}}{16 \pi^{2}}\left[\left(\frac{7}{3}-\frac{\alpha}{2}\right) \ln \left(\frac{p^{2}}{\kappa^{2}}\right)+\pi_{0}(\alpha)\right]\right\} \\
& \times \delta^{a b}\left(p^{2} g_{\mu \nu}-p_{\mu} p_{\nu}\right) .
\end{aligned}
$$

Next we move on to the ghost self-energy and the $\bar{c} A c$-vertex. The regularized ghost self-energy at one loop $\Omega^{a b}(p, A)$ receives contributions from only the diagram in Fig. 3 . This diagram is finite by power counting and, apart from the external momentum $p^{\mu}$, only depends on the mass $A$. To evaluate its limit $\Lambda \rightarrow \infty$, we use the same techniques as for the vacuum polarization tensor and obtain

$$
\Omega^{(1) a b}(p, \Lambda)=\frac{g^{2} c_{V}}{16 \pi^{2}}\left[\frac{3-\alpha}{4} \ln \left(\frac{p^{2}}{\Lambda^{2}}\right)+\omega_{0}(\alpha)\right] \delta^{a b} p^{2} .
$$

This gives for $\tilde{z_{2}}(\alpha)$ in Eqs. (4.4), (4.5) the value

$$
\tilde{z}_{2}(\alpha)=\frac{3-\alpha}{4} \text {. }
$$




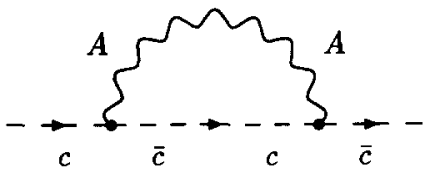

Fig. 3. One-loop regularization of the ghost self-energy.
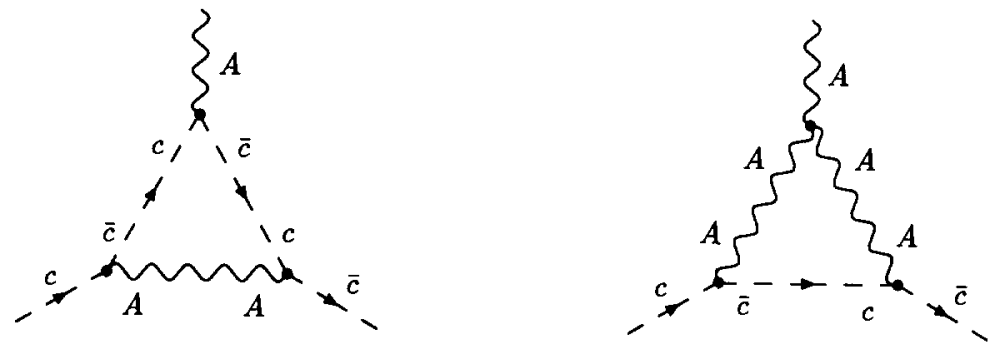

Fig. 4. One-loop regularization of the ghost-ghost-vertex.

As concerns the $\bar{c} A c$-vertex, at one loop it receives contributions from the two diagrams in Fig. 4. These diagrams are finite by power counting and depend on the external momenta $k^{\mu}$ and $p^{\mu}$ and the mass $A$. Using the same techniques as in Appendix B, it is not difficult to see that

$$
V_{\mu}^{(1) a b c}(k, p, \Lambda)=-i g f^{a b c} \frac{g^{2} c_{V}}{16 \pi^{2}}\left[-\frac{\alpha}{2} \ln \left(\frac{p^{2}}{\Lambda^{2}}\right) p_{\mu}+\alpha V_{\mu}^{\mathrm{fin}}(k, p, \alpha)\right]
$$

as $A$ goes to infinity. This yields

$$
\tilde{z}_{3}=-\frac{\alpha}{2} \text {. }
$$

Substituting the values obtained for $z_{2}(\alpha), \tilde{z}_{2}(\alpha)$ and $\tilde{z}_{3}(\alpha)$ in the expressions for the beta functions and the anomalous dimensions above, we have

$$
\begin{aligned}
& \beta(g)=-\frac{23}{6} \frac{g^{3} c_{V}}{16 \pi^{2}}+O\left(g^{4}\right), \quad \gamma_{A}(g)=\left(\frac{14}{3}-\alpha\right) \frac{g^{2} c_{V}}{16 \pi^{2}}+O\left(g^{3}\right) \\
& \gamma_{c}(g)+\gamma_{\bar{c}}(g)=(3-\alpha) \frac{g^{2} c_{V}}{16 \pi^{2}}+O\left(g^{3}\right), \quad \delta(g)=O\left(g^{2}\right) .
\end{aligned}
$$

The results for the beta function and the anomalous dimension of the gauge field are in disagreement with the well known results

$$
\beta(g)=-\frac{11}{3} \frac{g^{3} c_{V}}{16 \pi^{2}}+O\left(g^{4}\right), \quad \gamma_{A}(g)=\left(\frac{13}{3}-\alpha\right) \frac{g^{2} c_{V}}{16 \pi^{2}}+O\left(g^{3}\right) .
$$

We recall that for unitary theories the one and two-loop coefficients in the beta function and the one-loop coefficient of the anomalous dimensions are universal quantities that do 
not depend on the regularization method nor renormalization scheme used to compute them. In view of this, we have to conclude that the regularization prescription under consideration does not yield a consistent renormalized Yang-Mills theory, hence that it must be discarded as a candidate to regularize non-abelian gauge theories.

\section{Non-physical radiative corrections from Pauli-Villars determinants}

The beta function at one loop is built up with radiative corrections of type $\ln \left(p^{2} / \kappa^{2}\right)$. Hence, the fact that the result in Eq. (4.17) is not correct indicates that the regularization method generates somewhere non-local logarithmic radiative corrections which should not be there. To find these corrections and their origin we proceed as follows.

Suppose that instead of the regularization prescription of Faddeev and Slavnov we use dimensional regularization or a plain cut-off. Then the vacuum polarization tensor, the ghost self-energy and the ghost-vertex take after renormalization the form

$$
\begin{aligned}
& \Pi_{R \mu \nu}^{\prime a b}(p, \kappa, g, \alpha)=-\left\{1+\frac{g^{2} c_{V}}{16 \pi^{2}}\left[\left(\frac{13}{6}-\frac{\alpha}{2}\right) \ln \left(\frac{p^{2}}{\kappa^{2}}\right)+\pi_{0}(\alpha)\right]\right\} \delta^{a b}\left(p^{2} g_{\mu \nu}-p_{\mu} p_{\nu}\right) \\
& \Omega_{R}^{\prime a b}(p, \kappa, g, \alpha)=\left\{1+\frac{g^{2} c_{V}}{16 \pi^{2}}\left[\frac{3-\alpha}{4} \ln \left(\frac{p^{2}}{\kappa^{2}}\right)+\omega_{0}(\alpha)\right]\right\} \delta^{a b} p^{2} \\
& V_{R}^{\prime} \mu^{a b c}(k, p, \kappa, g, \alpha)=-i g f^{a b c}\left\{\left[1-\frac{g^{2} c_{V}}{16 \pi^{2}} \frac{\alpha}{2} \ln \left(\frac{p^{2}}{\kappa^{2}}\right)\right] p_{\mu}+\frac{g^{2} c_{V}}{16 \pi^{2}} \alpha V_{\mu}^{\mathrm{fin}}(k, p, \alpha)\right\} .
\end{aligned}
$$

From general theorems of renormalization theory, we know that two renormalized series for a 1PI Green function that agree up to order $\hbar^{n-1}$ can differ at order $\hbar^{n}$ by at most a local term. It happens however that the renormalized series for the vacuum polarization tensor in Eqs. (4.14) and (5.1) agree at tree level but differ at one loop by a nonlocal term. The question that arises then is whether Eqs. (4.14) and (5.1) correspond to different but consistent quantum Yang-Mills theories. To answer this question, we remind the reader that in Minkowski space the renormalized vacuum polarization tensor takes up to one loop the form

$$
\begin{aligned}
\Pi_{M \mu \nu}^{a b}(p, \kappa, g, \alpha)= & -\left\{1+\frac{g^{2} c_{V}}{16 \pi^{2}}\left[c(\alpha) \ln \left(\frac{-p^{2}+i 0^{+}}{\kappa^{2}}\right)+\pi_{0}(\alpha)\right]\right\} \\
& \times \delta^{a b}\left(p^{2} g_{\mu \nu}-p_{\mu} p_{\nu}\right) .
\end{aligned}
$$

The coefficient $c(\alpha)$ is uniquely determined by the imaginary part of $\Pi_{M \mu \nu}^{a b}$,

$$
\operatorname{Im} \Pi_{M \mu \nu}^{a b}(p, \kappa, g, \alpha)=-c(\alpha) \frac{g^{2} c V}{16 \pi^{2}} \theta\left(p^{2}\right)\left(p^{2} g_{\mu \nu}-p_{\mu} p_{\nu}\right)
$$

But the imaginary part of $\Pi_{M}^{a b}$ can be computed by means of Cutkosky rules as 


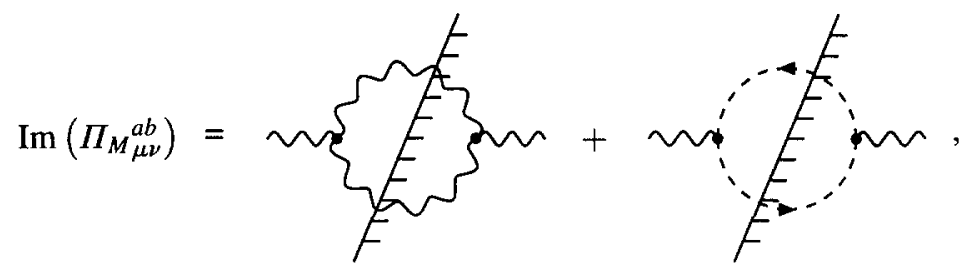

where the cut propagators and the vertices are the cut propagators and vertices of unregularized Yang-Mills theory. The cut-diagrams on the right-hand side are very easy to calculate in the Feynman gauge. Doing so and using Eq. $(5.2)$, we get $c(1)=5 / 3$. This selects the vacuum polarization tensor in Eq. (5.1) and disregards that in Eq. (4.14). It also shows that the vacuum polarization tensor as computed with higher covariant derivatives and Pauli-Villars fields contains unphysical logarithmic radiative corrections.

To pin-point the origin of these unphysical radiatives corrections, we consider the dimensionally regularized versions of the diagrams that contribute to the vacuum polarization tensor at finite $A, M_{j}, m_{i}$ and compute their limit $D \rightarrow 4$. Two types of contributions arise: contributions finite at $D=4$, and contributions singular at $D=4$. In the first ones, we set $D=4$ and take the limit $A, M_{j}, m_{i} \rightarrow \infty$. In the second ones, we leave $D$ arbitrary and keep the masses $\Lambda, M_{j}$ and $m_{i}$ finite. Proceeding in this way we obtain the results given in Appendix B. It follows from them that only diagrams (1a), (1e), (2a) and (2b) give $\ln \left(p^{2}\right)$-contributions. The $\ln \left(p^{2}\right)$-contribution from diagrams (1a) and (1e) can be read off from Eqs. (B.4) and (B.6):

$$
(1 \mathrm{a})+(1 \mathrm{e}) \rightarrow-\frac{g^{2} c_{V}}{16 \pi^{2}} \delta^{a b}\left(\frac{13}{6}-\frac{\alpha}{2}\right) \ln \left(p^{2}\right)\left(p^{2} g_{\mu \nu}-p_{\mu} p_{\nu}\right)
$$

As for diagrams (2a) and (2b), their $\ln \left(p^{2}\right)$-contribution is [see Eqs. (B.8), (B.16), (B.21) and (B.25)]

$$
(2 \mathrm{a})+(2 \mathrm{~b}) \rightarrow-\frac{g^{2} c_{V}}{16 \pi^{2}} \delta^{a b} \frac{1}{6} \ln \left(p^{2}\right)\left(p^{2} g_{\mu \nu}-p_{\mu} p_{\nu}\right)
$$

It thus becomes clear that diagrams (1a) and (1e) give by themselves the correct non-local part of the vacuum polarization tensor, whereas diagrams (2a) and (2b) are responsible for the unwanted logarithmic corrections. The problem is that without diagrams (2a) and (2b) the regularized vacuum polarization tensor is not finite nor transverse. This, together with the observation that diagrams (2a) and (2b) are generated by the determinants $\operatorname{det} \mathbb{A}_{j}^{1}$, leads to the conclusion that the very same Pauli-Villars determinants that regularize divergences also generate unphysical non-local logarithmic radiative corrections.

The determinant $\operatorname{det} \mathbb{A}_{j}^{1}$ has a mass $A$ hidden in it. One would like to know if this dependence on $A$ plays any part in the generation of the unphysical logarithmic radiative corrections. To see if this is the case, we replace the action $S_{A}$ in the definition of $\operatorname{det} \mathbb{A}_{j}^{1}$ with the Yang-Mills gauge-fixed action $S$ in Eq. (2.1) and re-compute the vacuum polarization tensor. The Feynman rules are now different and can be obtained from those in Appendix A by simply setting $A$ to infinity. The diagrams that make 
the vacuum polarization tensor at one loop are the same as in Figs. 1 and 2, with the difference that now they are constructed with different Feynman rules. To be on the safe side, and since Pauli-Villars determinants may not prove sufficient to regularize all UV divergences, we introduce in addition dimensional regularization. Using Eqs. (2.17) and (2.20), and after some lengthy but straightforward calculations, we obtain

$$
\Pi_{\mu \nu}^{(1) a b}\left(p, M_{j}, m_{i}, \varepsilon, \mu\right)=-\frac{g^{2} c_{V}}{16 \pi^{2}} \delta^{a b} \Pi\left(p, M_{j}, m_{i}\right)\left(p^{2} g_{\mu \nu}-p_{\mu} p_{\nu}\right),
$$

where $\Pi\left(p, M_{j}, m_{i}, \varepsilon, \mu\right)$ is given by

$$
\begin{aligned}
\Pi\left(p, M_{j}, m_{i}, \varepsilon, \mu\right)= & -\frac{3+\alpha}{2} L\left(p^{2}\right)-\frac{7}{2} \sum_{j=1}^{J} \alpha_{j} \ln \left(\frac{p^{2}}{M_{j}^{2}}\right) \\
& -\frac{1}{3} \sum_{i=1}^{J} \gamma_{i} \ln \left(\frac{p^{2}}{m_{i}^{2}}\right)+\pi_{0}\left(\alpha, M_{j}, m_{i}\right) .
\end{aligned}
$$

Here $L\left(z^{2}\right)$ denotes

$$
L\left(z^{2}\right)=\frac{1}{\varepsilon}+\ln \left(\frac{z^{2}}{\mu^{2}}\right) \quad D=4+2 \varepsilon
$$

and $\pi_{0}\left(\alpha, M_{j}, m_{i}\right)$ collects local contributions which are finite at $D=4$ and either vanish or remain finite as $M_{j}, m_{i} \rightarrow \infty$. The coefficient in front of $\ln \left(p^{2}\right)$ is $\frac{7}{3}-\alpha / 2$, the same as in Eqs. (4.2) and (4.13). Furthermore, although we have not given here partial results, it happens again that only diagrams (1a), (1e), (2a) and (2b) generate $\ln \left(p^{2}\right)$-contributions and that the latter contributions have the same form as in Eqs. (5.3) and (5.4). Hence the origin of the unphysical radiative corrections is not the $A$ hidden in $S_{A}$ in but the structure of the determinants $\operatorname{det} \mathbb{A}_{j}^{1}$.

Let us further argue that the non-local logarithmic contribution arising from diagrams (2a) and (2b) can not be set to zero by sending the masses $A$ and $M_{j}$ to infinity in a clever way. Suppose we take the diagrams in Figs. 1 and 2 and proceed as follows:

(i) Set the masses $A, M_{j}$ and $m_{i}$ to infinity in the Feynman integrands and keep only those diagrams whose integrands do not vanish;

(ii) Wick rotate to Minkowski's momentum space the surviving diagrams;

(iii) Compute the imaginary part of the analytically continued diagrams by means of Cutkosky rules (for a general reference on cut diagrams see Ref. [13]).

Then, it is not difficult to see that only diagrams (1a), (1e), (2a) and (2b) develop an imaginary part and that in the Feynman gauge the latter takes the form

$$
\begin{aligned}
& \operatorname{Im}[(1 \mathrm{a})+(1 \mathrm{e})]=-\frac{5}{3} \frac{g^{2} c_{V}}{16 \pi} \theta\left(p^{2}\right)\left(p^{2} g_{\mu \nu}-p_{\mu} p_{\nu}\right), \\
& \operatorname{Im}[(2 \mathrm{a})+(2 \mathrm{~b})]=-\frac{1}{6} \frac{g^{2} c_{V}}{16 \pi} \theta\left(p^{2}\right)\left(p^{2} g_{\mu \nu}-p_{\mu} p_{\nu}\right) .
\end{aligned}
$$

So diagrams (1a) and (1e) keep giving physical logarithmic radiative corrections and diagrams (2a) and (2b) keep giving unphysical logarithmic radiative corrections. The 
formal manipulations in steps (i) -(iii) are the standard manipulations used to compute the imaginary part of vacuum polarization tensor in QED [14] and rely on the following observation. In Minkowski space only diagrams with the topology of diagram (1a) develop an imaginary part at one loop. When the regulators are switched off, these diagrams are given by integrals of the form

$$
\int \frac{\mathrm{d}^{4} q}{(2 \pi)^{4}} \frac{P_{\mu \nu}(q, p)}{\left(q^{2}+i 0^{+}\right)\left[(q+p)^{2}+i 0^{+}\right]},
$$

where $P_{\mu \nu}(q, p)$ is some polynomial in the momenta $q^{\mu}$ and $p^{\mu}$. Rigorously speaking, this integral is a distribution which is not well defined. The point is that it can be properly defined without touching its imaginary part; this is what regularization and renormalization is all about. Therefore, the imaginary part of the integral before and after regularization and renormalization should be the same. Note, as a matter of fact, that for the $\left(\Lambda, M_{j}\right)$ paths considered in this paper, this simple way to proceed gives after Wick rotation to Euclidean space the same results as the explicit computations of Appendix B. It thus seems that the process in steps (i)-(iii) is correct if one is interested only in the imaginary part of the vacuum polarization tensor in Minkowski space, equivalently the non-local part in Euclidean space. Furthermore, since steps (i)(iii) do not depend on the path in $\left(\Lambda, M_{j}\right)$-space followed to send $\Lambda$ and $M_{j}$ to infinity, one would expect the renormalized vacuum polarization tensor to have the form in Eqs. (4.2) and (4.13) for all $\left(\Lambda, M_{j}\right)$ paths.

The question that arises now is whether it is possible to amend the regularization method so as to get rid of the unphysical non-local radiative corrections. This is possible but at the cost of losing manifest BRS invariance. To see this, let us assume that we take the gauge non-invariant version $r=0$ of the Pauli-Villars determinants, and let us take for concreteness the $\left(\Lambda, M_{j}\right)$ path in Eq. (4.9) with $\theta_{j}=\infty$. Now there are only six Feynman diagrams that contribute to the vacuum polarization tensor, those in Fig. 1. Evaluating them in dimensional regularization and taking the limit $\Lambda, M_{j}, m_{i} \rightarrow \infty$ in those contributions which are finite at $D=4$, we obtain (see Appendix B for partial results)

$$
\begin{aligned}
& \left.\Pi_{\mu \nu}^{(1) a b}\left(p, \Lambda, M_{j}, m_{i}\right)\right|_{r=0} \\
& \quad=\frac{g^{2} c_{V}}{16 \pi^{2}} \delta^{a b}\left\{-\frac{\pi \sqrt{3}}{4} \sum_{j=1}^{J} \alpha_{j} M_{j}^{2 / 3} \Lambda^{4 / 3} g_{\mu \nu}-\frac{1}{2} \sum_{i=1}^{I} \gamma_{i} \ln \left(\frac{m_{i}^{2}}{\mu^{2}}\right) m_{i}^{2} g_{\mu \nu}\right. \\
& \quad-\left[\left(\frac{31}{18}+\frac{\alpha}{2}\right) \ln \left(\frac{p^{2}}{\Lambda^{2}}\right)+\frac{143}{36} \sum_{i=1}^{I} \alpha_{j} \ln \left(\frac{p^{2}}{M_{j}^{2}}\right)-\frac{1}{12} \sum_{i=1}^{I} \gamma_{i} \ln \left(\frac{p^{2}}{m_{i}^{2}}\right)+\text { const }\right] p^{2} g_{\mu \nu} \\
& \left.+\left[\left(\frac{17}{9}+\frac{\alpha}{2}\right) \ln \left(\frac{p^{2}}{\Lambda^{2}}\right)+\frac{35}{9} \sum_{i=1}^{I} \alpha_{j} \ln \left(\frac{p^{2}}{M_{j}^{2}}\right)+\frac{1}{6} \sum_{i=1}^{I} \gamma_{i} \ln \left(\frac{p^{2}}{m_{i}^{2}}\right)+\text { const }\right] p_{\mu} p_{\nu}\right\} .
\end{aligned}
$$


Here we have imposed conditions (2.17), (2.20) and (2.21), which we recall from Subsection 2.2.2 ensure regularization of divergences. The right-hand side in Eq. (5.6) is not transverse, hence BRS invariance does not hold at the regularized level. To end up with a transverse renormalized vacuum polarization tensor, we proceed as follows. We further restrict the Pauli-Villars masses and parameters to satisfy

$$
\sum_{j=1}^{J} \alpha_{j} M_{j}^{2 / 3}=0, \quad \sum_{i=1}^{I} \gamma_{i} m_{i}^{2} \ln \left(m_{i}^{2}\right)=0 .
$$

By doing this we get rid of the first two terms in Eq. (5.6). Next we introduce a renormalization mass scale $\kappa$ and perform a suitable subtraction. The fact that the coefficient in front of $\ln \left(p^{2}\right)$ in both the terms $p^{2} g_{\mu \nu}$ and $p_{\mu} p_{\nu}$ in Eq. (5.6) is the same and equal to $\frac{13}{6}-\alpha / 2$-compare with Eq. (5.1) -ensures that the renormalized vaccum polarization tensor will be transverse and will have the correct non-local part. If instead of $\left(A, M_{j}\right)$ paths with $\theta_{j}=\infty$ we take paths with $0 \leqslant \theta_{j}<\infty$ and use the results in Appendix $\mathrm{B}$, it is very easy to see that the same conclusion holds. The only difference is that to ensure transversality, we must replace the condition on the masses $M_{j}$ in Eq. (5.7) with

$$
\sum_{j=1}^{J} \alpha_{j} M_{j}^{2}=0 .
$$

It thus becomes clear that the terms introduced when $r=0$ is replaced with $r=1$ so as to have BRS invariance at the regularized level originate the unphysical non-local logarithmic corrections. Note in this regard that diagrams (2a) and (2b), the diagrams that originated the unphysical contributions, do not exist at all for $r=0$. The problem with taking $r=0$ is that manifest BRS is lost, thus becoming uninteresting.

\section{Conclusion}

In this paper we have addressed the question of whether higher covariant derivative Pauli-Villars regularization as proposed in Refs. [2,3] leads to a consistent renormalized Yang-Mills theory. The regularization prescription is described by the generating functional $Z_{1}[J, \chi, \eta, \bar{\eta}]$ in Eq. (2.12) and combines a higher covariant derivative term in the action with a set of Pauli-Villars determinants $\operatorname{det} \mathbb{A}_{j}^{1}$ and $\operatorname{det} \mathbb{C}_{i}^{1}$. The main results of our investigation can be summarized in the following three points:

(1) There are certain subtleties regarding the regularization mechanism that have gone unnoticed in the previous literature and that reveal that, even after imposing suitable Pauli-Villars conditions, the regularized Green functions are not finite in the power counting sense advertized in Ref. [3]. This has been explicitly realized in Subsection 2.2.2, where it is shown that checking that the regularized vacuum polarization tensor is finite for finite values of the regulators requires introducing a further regulator $\mathcal{R}$. This gives lie to the claim $[2,3]$ that the functional $Z_{1}[J, \chi, \eta, \bar{\eta}]$ constitutes by itself a regularized expression for the Yang-Mills path integral. Yet, since the non-local part 
of the regularized Green functions is the same for all admissible regulators $\mathcal{R}$, one can think of using the functional $Z_{1}[J, \chi, \eta, \bar{\eta}]$ to understand physical properties like e.g. unitarity.

(2) The regularization prescription does lead to a renormalized theory consistent with gauge invariance. This settles down some controversy in the previous literature $[8,9]$.

(3) The Pauli-Villars determinants on which the regularization method is based generate unphysical non-local logarithmic radiative corrections. These corrections, being non-local, survive renormalization and give an inconsistent renormalized Yang-Mills theory. In particular, the beta function of the resulting renormalized theory has a one-loop coefficient equal to $-23 / 6$, in contradiction with general results from renormalization theory that state that it should be $-11 / 3$. We expect these logarithmic unphysical corrections to break unitarity in Minkowski spacetime when the theory is coupled to matter. In fact, if unitarity is formulated in the sense of van Dam and Veltman [15], our discussion in Section 5 already shows that this is the case. We have also seen that it is possible to modify the Pauli-Villars determinants so as to get rid of the unwanted radiative corrections, but this is at the expense of losing gauge invariance at the regularized level.

In view of all this we have to conclude that higher covariant derivative Pauli-Villars regulators do not provide an admissible gauge invariant regularization method.

\section{Acknowledgements}

The authors are grateful to G. 't Hooft, J.C. Taylor and M. Veltman for discussions. FRR was supported by FOM, The Netherlands. Partial support from CICyT, Spain is also acknowledged.

\section{Appendix A. Feynman rules}

Here we list the Feynman rules corresponding to the functional $Z_{r}[J, \chi, \eta, \bar{\eta}]$ in Eq. (2.12). The propagators are given by

$$
\begin{aligned}
& \sim \sim \overrightarrow{A_{\mu}^{a}} \sim \sim_{A_{\nu}^{b}}^{\stackrel{p}{\vec{n}}}=\frac{\delta^{a b} \Lambda^{4}}{p^{4}\left(p^{4}+\Lambda^{4}\right)}\left[p^{2} g_{\mu \nu}-p_{\mu} p_{\nu}\left(1-\frac{\alpha A^{4}}{p^{4}+\Lambda^{4}}\right)\right] \\
& \stackrel{\sim}{\bullet} \sim \sim_{A^{b}}^{b}=-i \delta^{a b} \frac{p_{\mu}}{p^{2}} \\
& \underset{\bar{c}^{a}}{\bullet-\underset{c^{b}}{\rightarrow}} \stackrel{p}{\rightarrow}=-\frac{\delta^{a b}}{p^{2}}
\end{aligned}
$$


572

C.P. Martin, F. Ruiz Ruiz/Nuclear Physics B 436 (1995) 545-581

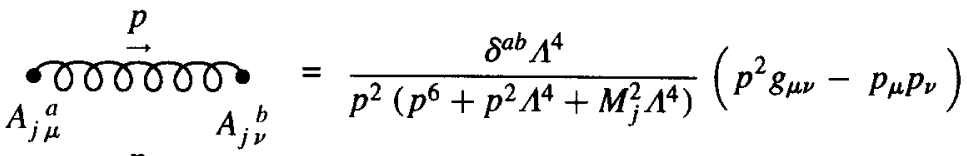

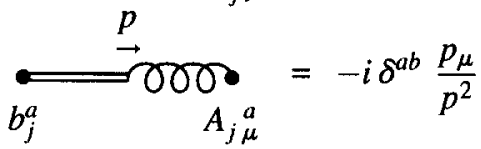

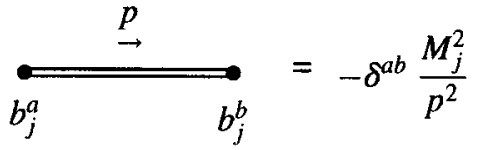

$$
\begin{aligned}
& \begin{array}{c}
p \\
\bar{c}_{i}^{a} \\
c_{i}^{b}
\end{array}=-\frac{\delta^{a b}}{p^{2}+m_{i}^{2}},
\end{aligned}
$$

where $p^{4}$ stands for $\left(p^{2}\right)^{2}$. Note that the auxiliary field $b_{j}^{a}$ propagates with propagator proportional to $M_{j}^{2}$, whereas the field $b^{a}$ does not propagate at all. As for the vertices, we group them according to their order in the coupling constant $g$. Vertices of order $g$ are given by

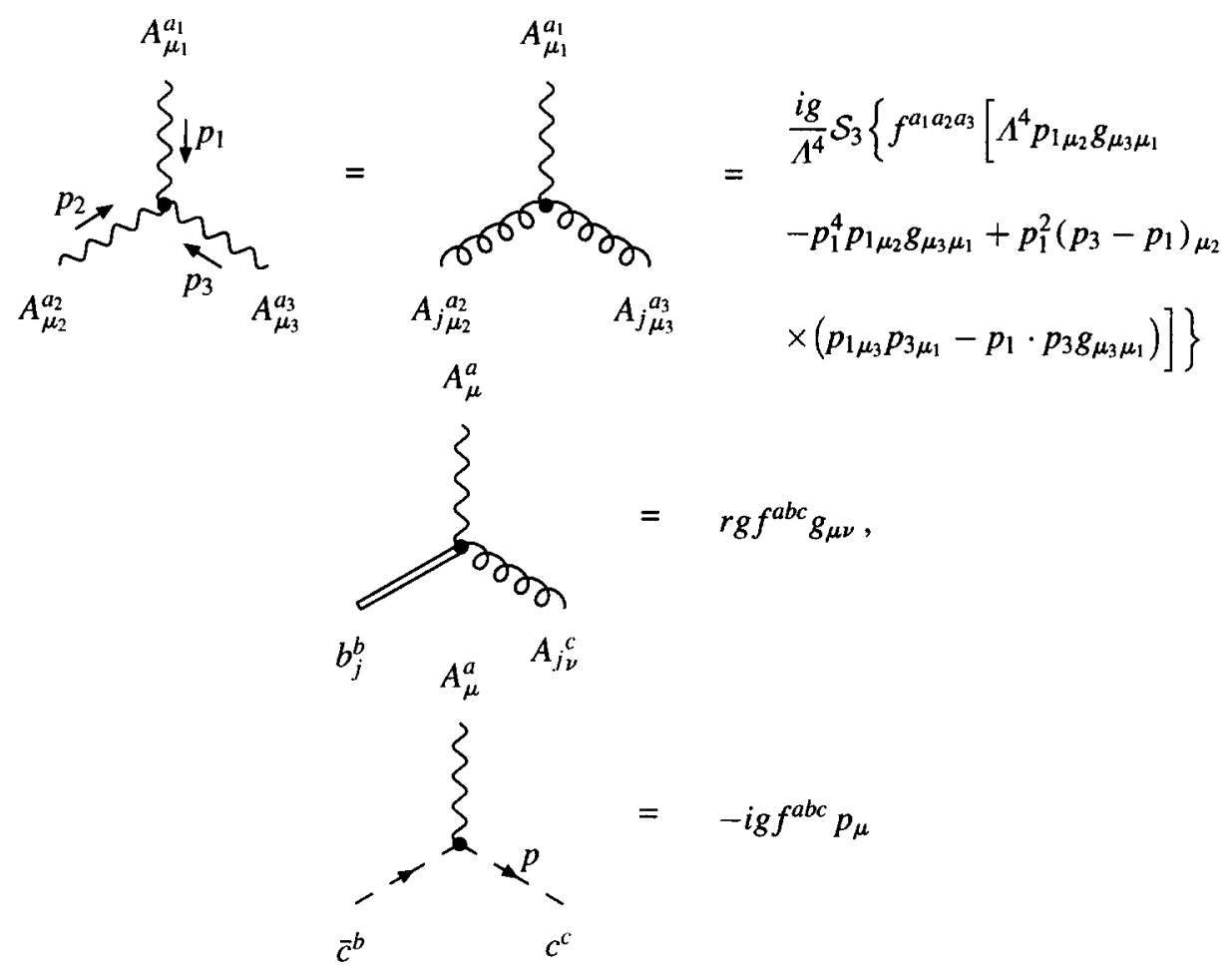




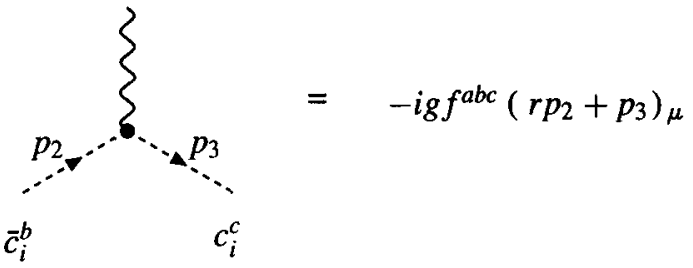

where $\mathcal{S}_{3}$ is the symmetrization operator with respect to the indices 1,2 and 3 , and $r$ is the parameter distinguishing gauge non-invariant $(r=0)$ from gauge invariant $(r=1)$ Pauli-Villars determinants. Vertices of order $g^{2}$ have the form

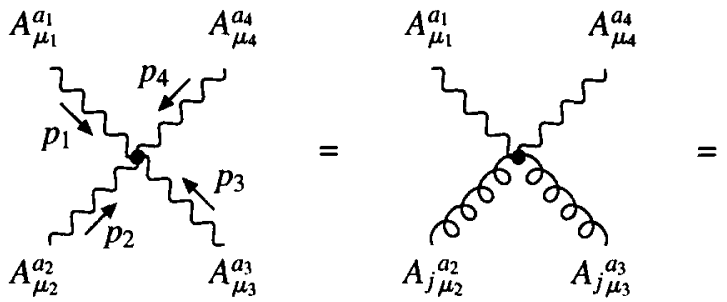

$$
\begin{aligned}
= & -\frac{g^{2}}{\Lambda^{4}} \mathcal{S}_{4}\left\{f ^ { a _ { 1 } a _ { 2 } b } f ^ { a _ { 3 } a _ { 4 } b } \left[\Lambda^{4} g_{\mu_{1} \mu_{3}} g_{\mu_{2} \mu_{4}}+\left(p_{1}+p_{2}\right)^{4} g_{\mu_{1} \mu_{3}} g_{\mu_{2} \mu_{4}}\right.\right. \\
& +4 p_{1}^{2} g_{\mu_{2} \mu_{3}}\left(p_{4 \mu_{1}} p_{1 \mu_{4}}+p_{1} \cdot p_{4} g_{\mu_{1} \mu_{4}}\right)-4 p_{1}^{2} g_{\mu_{1} \mu_{4}}\left(2 p_{1}+p_{2}\right)_{\mu_{2}} p_{1 \mu_{3}} \\
& +2 p_{1 \mu_{1}} p_{3 \mu_{3}}\left(p_{2} \cdot p_{4} g_{\mu_{2} \mu_{4}}-p_{2 \mu_{4}} p_{4 \mu_{2}}\right)+4\left(p_{1}+p_{2}\right)^{2} g_{\mu_{2} \mu_{4}} p_{4 \mu_{1}}\left(p_{3}+2 p_{4}\right)_{\mu_{3}} \\
& \left.\left.+8\left(p_{1}+p_{2}\right)_{\mu_{1}} p_{4 \mu_{3}}\left(p_{2} \cdot p_{4} g_{\mu_{2} \mu_{4}}-p_{2 \mu_{4}} p_{4 \mu_{2}}\right)\right]\right\}
\end{aligned}
$$

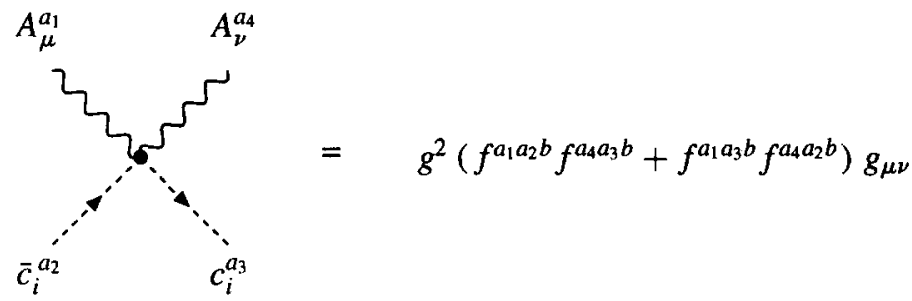

where $\mathcal{S}_{4}$ is the symmetrization operator with respect to the indices $1,2,3$ and 4 . The action $S_{A}$ and det $\mathbb{A}_{j}$ also contain vertices of higher order in $g$. However, these only enter two and higher-loop 1PI diagrams, so we do not need them here. 


\section{Appendix B. Computing the limit $\Lambda, M_{j}, m_{i} \rightarrow \infty$}

In this Appendix we give the details of the calculations leading to the results of Sections 4 and 5. We start presenting a large mass vanishing theorem which simplifies very much the computation of the large mass limit of the Feynman integrals we are interested in. Let us denote by $p_{1}^{\mu}, \ldots, p_{E}^{\mu}$ some external momenta lying in a bounded subdomain of $\mathbb{R}^{n}$ and let $m>0$ be a mass. Consider an $L$-loop integral of the form

$$
I(p, m)=m^{\eta} \int \mathrm{d}^{n} q_{1} \ldots \mathrm{d}^{n} q_{L} \frac{M(q)}{\prod_{i}\left[\left(k_{i}^{2}\right)^{n_{i}}+m_{i}^{2 n_{i}}\right]^{l_{i}}},
$$

where $\eta$ is an arbitrary real number and

$$
\begin{aligned}
M(q) & =\text { monomial in the components of } q_{1}^{\mu}, \ldots, q_{L}^{\mu} ; \\
k_{i}^{\mu} & =\text { linear combination of } p_{1}^{\mu}, \ldots, p_{E}^{\mu} \text { and } q_{1}^{\mu}, \ldots, q_{L}^{\mu} ; \\
m_{i} & =\text { either } 0 \text { or } m ; \\
n_{i}, l_{i} & =\text { positive integers } .
\end{aligned}
$$

Given a subintegral $J$ of $I(p, m)$, we call $\underline{\omega}_{J}$ to its infrared degree at vanishing external momenta and denote by $\underline{\omega}_{\min }=\min _{J}\left\{0, \underline{\omega}_{J}\right\}$ the minimum of zero and the infrared degrees $\underline{\omega}_{J}$ of all the subintegrals $J$ of $I(p, m)$ including $I(p, m)$ itself. Then, the following theorem holds:

$m$-Theorem. If the integral $I(p, m)$ is absolutely convergent at non-exceptional external momenta, and if its mass dimension $d$ and $\underline{\omega}_{\min }$ defined above satisfy $d-\underline{\omega}_{\min }<0$, then $I(p, m) \rightarrow 0$ as $m \rightarrow \infty$.

This is a trivial generalization of the $m$-theorem in Ref. [16], to where we refer for the proof. Armed with this theorem, we proceed to derive the results in Eqs. (4.10)-(4.12) for the vacuum polarization tensor.

We start by recalling that the vacuum polarization tensor receives contributions at one loop from the eleven Feynman diagrams in Figs. 1 and 2. To compute these diagrams, we first replace each diagram by its dimensionally regularized version, then make our computations using dimensional regularization techniques, and finally take the limit $D \rightarrow 4$. To see that this way to proceed is legitimate and does not introduce ambiguities of any type, we recall the following property of dimensionally regularized integrals [17]: If $I$ is an absolutely convergent integral defined in four dimensions, and if $I(D)$ denotes the corresponding dimensionally regularized integral, then

$$
I=\lim _{D \rightarrow 4} I(D) .
$$

This property, together with the fact that the sums $\sigma_{1}, \sigma_{2}$ and $\sigma_{4}^{1}=(2 a)$ in Section 2 are given by sums of finite by power counting integrals, implies that $\sigma_{1}, \sigma_{2}$ and $\sigma_{4}^{1}$ can be computed as explained. In the case of $\sigma_{3}$, proving finiteness required an additional regulator $\mathcal{R}$. Our approach here takes this regulator to be dimensional regularization. It 
will of course happen that isolated diagrams will have poles at $D=4$, but these poles will cancel after summing over diagrams and imposing conditions (2.17) and (2.20).

We first compute those diagrams which depend at most on one mass. To illustrate how to proceed, we take diagram (1a) as an example and look at $\alpha=0$ contributions. With the labeling of internal momenta in Fig. 1a, and dropping from the notation the delta $\delta^{a b}$, the most general dimensionally regularized integral arising from diagram (1a) with $\alpha=0$ will have the form

$$
I_{\mu \nu}^{\mathrm{la}}\left(D ; n_{q}, n_{k}, n_{A}\right)=\frac{g^{2} c_{V}}{\mu^{D-4}} \int \frac{\mathrm{d}^{D} q}{(2 \pi)^{D}} \frac{\left(\Lambda^{n_{A}} q^{n_{q}} k^{n_{k}} p^{n_{p}}\right)_{\mu \nu}}{q^{4}\left(q^{4}+\Lambda^{4}\right) k^{4}\left(k^{4}+\Lambda^{4}\right)} .
$$

The numerator in the integrand is a monomial in $\Lambda$ and in the components of $q^{\mu}, k^{\mu}=$ $q^{\mu}+p^{\mu}$ and $p^{\mu}$ of degrees $n_{A}, n_{q}, n_{k}$ and $n_{p}$, respectively. Since the vacuum polarization tensor has mass dimension two, the $n$ 's satisfy $n_{A}+n_{q}+n_{k}+n_{p}=14$. Furthermore, since the propagator of the gauge field carries two powers of the momentum in the numerator, we have $n_{q}, n_{k} \geqslant 2$, thus ensuring IR convergence. If $n_{q}+n_{k}<12$, the integral $I_{\mu \nu}^{\mathrm{a} a}\left(D ; n_{q}, n_{k}, n_{A}\right)$ is absolutely convergent by power counting at $D=4$. In this case we can set $D=4$ in Eq. (B.2) and move on to computing its large- $\Lambda$ limit. To do the latter, we use the $m$-theorem above. It is trivial to see that the theorem sates in this case that

$$
\left.\begin{array}{r}
n_{q}+n_{k}+n_{\Lambda}<12 \\
n_{k}+n_{\Lambda}<8
\end{array}\right\} \Rightarrow \lim _{\Lambda \rightarrow \infty} I_{\mu \nu}^{1 \mathrm{a}}\left(4 ; n_{q}, n_{k}, n_{\Lambda}\right)=0 .
$$

If $n_{q}+n_{k}+n_{A} \geqslant 12$ and/or $n_{k}+n_{A} \geqslant 8$, we proceed as follows. Take e.g. the integral

$$
J_{\mu \nu}^{\mathrm{la}}(D)=\frac{g^{2} c_{V}}{\mu^{D-4}} \int \frac{\mathrm{d}^{D} q}{(2 \pi)^{D}} \frac{q^{10} k^{2} p_{\mu} q_{\nu}}{q^{4}\left(q^{4}+\Lambda^{4}\right) k^{4}\left(k^{4}+\Lambda^{4}\right)} .
$$

Substituting $k^{\mu}$ by $q^{\mu}+p^{\mu}$ and performing some trivial algebra, we write

$$
J_{\mu \nu}^{1 a}(D)=J_{\mu \nu}^{\prime}(D)+J_{\mu \nu}^{\prime \prime}(D),
$$

where

$$
\begin{aligned}
& J_{\mu \nu}^{\prime}(D)=\frac{g^{2} c_{V}}{\mu^{D-4}} \int \frac{\mathrm{d}^{D} q}{(2 \pi)^{D}} \frac{q^{2}\left(q^{2}+2 p q\right) p_{\mu} q_{\nu}}{\left(q^{4}+\Lambda^{4}\right)\left[(q+p)^{4}+\Lambda^{4}\right]}, \\
& J_{\mu \nu}^{\prime \prime}(D)=-\frac{g^{2} c_{V}}{\mu^{D-4}} \int \frac{\mathrm{d}^{D} q}{(2 \pi)^{D}} \frac{q^{2}\left[p^{2} q^{2}-2 p q\left(p^{2}+2 p q\right)\right] p_{\mu} q_{\nu}}{\left(q^{4}+\Lambda^{4}\right)(q+p)^{2}\left[(q+p)^{4}+\Lambda^{4}\right]} .
\end{aligned}
$$

The integral $J_{\mu \nu}^{\prime \prime}(D)$ is absolutely convergent by power counting at $D=4$, so we can set $D=4$ in it. Doing so and using the $m$-theorem, we have

$$
\lim _{A \rightarrow \infty} J_{\mu \nu}^{\prime \prime}(4)=0 \text {. }
$$

To calculate $J_{\mu \nu}^{\prime}(D)$, we use Eq. (2.24) iteratively and whenever we come across an integral which is absolutely convergent by power counting at $D=4$, we set $D=4$ in it and use the $m$-theorem. After two or three iterations, we get

$$
J_{\mu \nu}^{\prime}(D)=\frac{g^{2} c_{\nu}}{\mu^{D-4}} \int \frac{\mathrm{d}^{D} q}{(2 \pi)^{D}}\left[\frac{q^{4} p_{\mu} q_{\nu}}{\left(q^{4}+\Lambda^{4}\right)^{2}}-\frac{2 q^{2}(p q) p_{\mu} q_{\nu}}{\left(q^{4}+\Lambda^{4}\right)^{2}}-\frac{4 q^{6}(p q) p_{\mu} q_{\nu}}{\left(q^{4}+\Lambda^{4}\right)^{3}}\right]+\ldots
$$


with the dots collecting integrals which are finite at $D=4$ and whose large- $\Lambda$ limit at $D=4$ vanishes. Using Eq. (B.27) below, it is now trivial to see that

$$
J_{\mu \nu}^{\prime}(D)=\frac{3}{2} \frac{g^{2} c_{V}}{16 \pi^{2}}\left[\frac{1}{\varepsilon}+\ln \left(\frac{A^{2}}{4 \pi \mu^{2}}\right)+\frac{1}{6}\right] p_{\mu} p_{\nu}
$$

as $\varepsilon \rightarrow 0$, where $D=4+2 \varepsilon$. In what follows we will denote by $v_{0}$ all mass-independent contributions which are finite at $D=4$. With this convention and the notation in Eq. (5.5), we finally write

$$
J_{\mu \nu}^{1 a}(D)=\frac{3}{2} \frac{g^{2} c_{V}}{16 \pi^{2}} L\left(\Lambda^{2}\right) p_{\mu} p_{\nu}+v_{0} .
$$

The contribution of any other integral (B.2) with $n_{q}+n_{k}+n_{\Lambda} \geqslant 12$ and/or $n_{k}+n_{A} \geqslant 8$ can be calculated in the same way. After some lengthy calculations and putting together all contributions ${ }^{3}$, we obtain for the complete diagram

$$
\text { (1a) } \begin{aligned}
= & \frac{g^{2} c_{\nu}}{16 \pi^{2}}\left\{\frac{3 \pi}{2}\left(\frac{\alpha}{4}-5\right) \Lambda^{2} g_{\mu \nu}-\left[\frac{11}{4} L\left(\Lambda^{2}\right)+\frac{1}{2}\left(\frac{25}{6}-\alpha\right) \ln \left(\frac{p^{2}}{\Lambda^{2}}\right)\right] p^{2} g_{\mu \nu}\right. \\
& \left.-\left[13 L\left(\Lambda^{2}\right)-\left(\frac{7}{3}-\frac{\alpha}{2}\right) \ln \left(\frac{p^{2}}{\Lambda^{2}}\right)\right] p_{\mu} p_{\nu}\right\}+v_{0} .
\end{aligned}
$$

Diagrams (1c), (1e), (1f), (2b) and (2e) are computed in the same way. For them we obtain

$$
\begin{aligned}
& \text { (1c) }=\frac{g^{2} c_{V}}{16 \pi^{2}}\left\{\frac{3 \pi}{2}\left(5-\frac{\alpha}{4}\right) \Lambda^{2} g_{\mu \nu}+\frac{4}{3} L\left(\Lambda^{2}\right)\left(11 p^{2} g_{\mu \nu}+p_{\mu} p_{\nu}\right)\right\}+v_{0}, \\
& \text { (le) }=-\frac{g^{2} c_{V}}{16 \pi^{2}} L\left(p^{2}\right)\left(\frac{1}{12} p^{2} g_{\mu \nu}+\frac{1}{6} p_{\mu} p_{\nu}\right)+v_{0} \\
& (1 \mathrm{f})=-\frac{g^{2} c_{V}}{16 \pi^{2}} \sum_{i=1}^{I} \gamma_{i} L\left(m_{i}^{2}\right)\left[\frac{(1+r)^{2}}{2} m_{i}^{2} g_{\mu \nu}+\frac{(1+r)^{2}}{12} p^{2} g_{\mu \nu}+\frac{1-4 r+r^{2}}{6} p_{\mu} p_{\nu}\right] \\
& +v_{0} \text {, } \\
& (2 \mathrm{~b})=\frac{g^{2} c_{V}}{16 \pi^{2}} r^{2} \sum_{j=1}^{J} \alpha_{j} L\left(p^{2}\right)\left(\frac{1}{12} p^{2} g_{\mu \nu}+\frac{1}{6} p_{\mu} p_{\nu}\right)+v_{0} \\
& \text { (2e) }=\frac{g^{2} c_{V}}{16 \pi^{2}} 2 r \sum_{i=1}^{I} \gamma_{i} L\left(m_{i}^{2}\right) m_{i}^{2} g_{\mu \nu}+v_{0}
\end{aligned}
$$

Let us next look at diagrams (1b), (1d), (2a), (2c) and (2d). They all depend on the masses $\Lambda$ and $M_{j}$ and their limit $\Lambda, M_{j} \rightarrow \infty$ is not unique but depends on the way in which we approach $A=M_{j}=\infty$. The three cases considered in Section 4 are

\footnotetext{
${ }^{3}$ The $\alpha$-dependent part of the diagram is built up of integrals finite by power counting at $D=4$ whose large- $A$ limit can be computed using similar methods.
} 
Case $\theta_{j}=\infty$

This corresponds to taking $M_{j} \rightarrow \infty$ ( $\Lambda<\infty$ fixed) first and then sending $\Lambda \rightarrow \infty$. Let us consider diagram ( $1 \mathrm{~b})$. The most general integral it gives rise to has the form

$$
I_{\mu \nu}^{\mathrm{lb}}\left(D ; n_{q}, n_{k}, n_{A}\right)=\sum_{j=1}^{J} \alpha_{j} \frac{g^{2} c_{V}}{\mu^{D-4}} \int \frac{\mathrm{d}^{D} q}{(2 \pi)^{D}} \frac{\left(\Lambda^{n_{A}} q^{n_{q}} k^{n_{k}} p^{n_{p}}\right)_{\mu \nu}}{q^{2} D_{j}(q) k^{4} D_{j}(k)},
$$

with

$$
D_{j}(q)=q^{6}+q^{2} \Lambda^{4}+M_{j}^{2} \Lambda^{4} .
$$

Again we have that $n_{A}+n_{q}+n_{k}+n_{p}=14$ and $n_{q}, n_{k} \geqslant 2$. If $n_{q}+n_{k}<12$, the integral is absolutely convergent by power counting at $D=4$ and we have that

$$
0 \leqslant\left|I_{\mu \nu}^{1 \mathrm{~b}}\left(4 ; n_{q}, n_{k}, n_{\Lambda}\right)\right| \leqslant g^{2} c_{V} \sum_{j=1}^{J}\left|\alpha_{j}\right| \int \frac{\mathrm{d}^{4} q}{(2 \pi)^{4}} \frac{\left(\Lambda^{n_{A}}|q|^{n_{q}}|k|^{n_{k}}|p|^{n_{p}}\right)_{\mu \nu}}{q^{2}\left(q^{6}+\lambda_{j}^{6}\right) k^{4}\left(k^{6}+\lambda_{j}^{6}\right)},
$$

where

$$
\lambda_{j}=M_{j}^{1 / 3} \Lambda^{2 / 3} .
$$

The $m$-theorem above implies that the the right-hand side in Eq. (B.11) goes to zero as $M_{j}$ approaches infinity and $\Lambda$ is kept fixed, thus yielding

$$
n_{q}+n_{k}<12 \Rightarrow \lim _{M_{j} \rightarrow \infty} I_{\mu \nu}^{\mathrm{lb}}\left(4 ; n_{q}, n_{k}, n_{A}\right)=0 .
$$

To illustrate how to proceed if $n_{q}+n_{q} \geqslant 12$, let us consider the integral [compare with Eq. (B.3)]

$$
J_{\mu \nu}^{\mathrm{lb}}(D)=\sum_{j=1}^{J} \alpha_{j} \frac{g^{2} c_{V}}{\mu^{D-4}} \int \frac{\mathrm{d}^{D} q}{(2 \pi)^{D}} \frac{q^{10} k^{2} p_{\mu} q_{\nu}}{q^{2} D_{j}(q) k^{2} D_{j}(k)} .
$$

Substituting $k^{\mu}$ by $q^{\mu}+p^{\mu}$ and using the identity

$$
\frac{1}{D_{j}(q+p)}=\frac{1}{D_{j}(q)}-\frac{D_{j}(q+p)-D_{j}(q)}{D_{j}(q) D_{j}(q+p)}
$$

and the $m$-theorem, we get

$$
J_{\mu \nu}^{\mathrm{lb}}(D)=\sum_{j=1}^{J} \alpha_{j} \frac{g^{2} c_{V}}{\mu^{D-4}} \int \frac{\mathrm{d}^{D} q}{(2 \pi)^{D}} \frac{q^{8} p_{\mu} q_{\nu}}{D_{j}^{2}(q)}\left[1-\frac{6 q^{4}(p q)}{D_{j}(q)}\right]+\ldots
$$

where the dots stand for integrals finite by power counting at $D=4$ that vanish at $D=4$ as $M_{j} \rightarrow \infty$ and $L a<\infty$ is kept fixed. Recalling now

$$
\frac{1}{D_{j}(q)}=\frac{1}{q^{6}+\lambda_{j}^{6}}-\frac{q^{2} \Lambda^{4}}{\left(q^{6}+\lambda_{j}^{6}\right) D_{j}(q)}
$$


and invoking the $m$-theorem again, we have

$$
J_{\mu \nu}^{\mathrm{lb}}(D)=\sum_{j=1}^{J} \alpha_{j} \frac{g^{2} c_{V}}{\mu^{D-4}} \int \frac{\mathrm{d}^{D} q}{(2 \pi)^{D}} \frac{q^{8} p_{\mu} q_{\nu}}{\left(q^{6}+\lambda_{j}^{6}\right)^{2}}\left[1-\frac{6 q^{4}(p q)}{\left(q^{6}+\lambda_{j}^{6}\right)}\right]+\ldots
$$

Performing the integral with the help of Eq. (B.28), we finally obtain

$$
J_{\mu \nu}^{1 \mathrm{~b}}(D)=\frac{3}{2} \frac{g^{2} c_{V}}{16 \pi^{2}} \sum_{j=1}^{J} \alpha_{j} L\left(\lambda_{j}^{2}\right) p_{\mu} p_{\nu}+v_{0},
$$

where the notation for $L\left(\lambda_{j}^{2}\right)$ is as in Eq. (5.5). Any other integral (B.10) with $n_{q}+n_{k} \geqslant 12$ can be evaluated in a similar fashion. After doing so and summing over all contributions to diagram (1b), we finally get

$$
\text { (1b) }=-\frac{g^{2} c_{V}}{16 \pi^{2}} \sum_{j=1}^{J} \alpha_{j}\left[4 \pi \sqrt{3} \lambda_{j}^{2} g_{\mu \nu}+L\left(\lambda_{j}^{2}\right)\left(\frac{11}{4} p^{2} g_{\mu \nu}+13 p_{\mu} p_{\nu}\right)\right]+v_{0} \text {. }
$$

Note that, provided one imposes the Pauli-Villars condition (2.17), the pole in $L\left(\lambda_{j}^{2}\right)$ cancels with the pole from $L\left(\Lambda^{2}\right)$ in Eq. (B.4) so as to give a finite partial sum $\sigma_{1}$.

The other diagrams are evaluated in the same way. For them we obtain

$$
\begin{aligned}
& (1 \mathrm{~d})=\frac{1}{3} \frac{g^{2} c_{V}}{16 \pi^{2}} \sum_{j=1}^{J} \alpha_{j}\left[\frac{23 \pi \sqrt{3}}{2} \lambda_{j}^{2} g_{\mu \nu}+4 L\left(\lambda_{j}^{2}\right)\left(11 p^{2} g_{\mu \nu}+p_{\mu} p_{\nu}\right)\right]+v_{0} \\
& (2 \mathrm{a})=\frac{r^{2}}{3} \frac{g^{2} c_{V}}{16 \pi^{2}} \sum_{j=1}^{J} \alpha_{j}\left[-\frac{\pi \sqrt{3}}{2} \lambda_{j}^{2} g_{\mu \nu}+\ln \left(\frac{p^{2}}{\lambda_{j}^{2}}\right)\left(\frac{1}{4} p^{2} g_{\mu \nu}-p_{\mu} p_{\nu}\right)\right]+v_{0} \\
& (2 \mathrm{c})+(2 \mathrm{~d})=\frac{r}{3} \frac{g^{2} c_{V}}{16 \pi^{2}} \sum_{j=1}^{J} \alpha_{j}\left[\pi \sqrt{3} \lambda_{j}^{2} g_{\mu \nu}+2 L\left(\lambda_{j}^{2}\right)\left(\frac{1}{4} p^{2} g_{\mu \nu}-p_{\mu} p_{\nu}\right)\right]+v_{0} .
\end{aligned}
$$

Taking $r=1$ and summing Eqs. (B.4)-(B.9) and (B.14)-(B.17), we obtain Eq. (4.10). Note that, in agreement with our discussion in Section 2, the partial sums $\sigma_{1}$, $\sigma_{2}, \sigma_{3}^{1}$ and $\sigma_{4}^{1}:=(2 \mathrm{a})$ are finite (no poles) provided condition (2.17) and (2.20) are met. Note also that contributions of type (masses) ${ }^{2} g_{\mu \nu}$ cancel in the vacuum polarization tensor upon summation. If we take $r=0$ instead, we recover the result in Eq. (5.6).

Case $\theta_{j}=0$

This corresponds to first taking $A \rightarrow \infty$ at fixed $M_{j}$ and then sending $M_{j}$ to $\infty$. Let us consider diagram ( $1 \mathrm{~b}$ ). As we already know, the most general dimensionally regularized Feynman integral arising from it has the form in Eq. (B.10). If $n_{q}+n_{k}<12$, the integral is finite by power counting at $D=4$. In this case, the inequality 


$$
0 \leqslant\left|I_{\mu \nu}^{1 \mathrm{~b}}\left(4 ; n_{q}, n_{k}, n_{\Lambda}\right)\right| \leqslant g^{2} c_{V} \sum_{j=1}^{J}\left|\alpha_{j}\right| \int \frac{\mathrm{d}^{4} q}{(2 \pi)^{4}} \frac{\left(\Lambda^{n_{A}}|q|^{n_{q}}|k|^{n_{k}}|p|^{n_{p}}\right)_{\mu \nu}}{q^{4}\left(q^{4}+\Lambda^{4}\right) k^{4}\left(k^{4}+\Lambda^{4}\right)}
$$

and the $m$-theorem yield

$$
\left.\begin{array}{r}
n_{q}+n_{k}+n_{\Lambda}<12 \\
n_{k}+n_{\Lambda}<8
\end{array}\right\} \Rightarrow \lim _{\Lambda \rightarrow \infty} I_{\mu \nu}^{\mathrm{lb}}\left(4 ; n_{q}, n_{k}, n_{\Lambda}\right)=0 .
$$

To explain how to proceed if $n_{q}+n_{k}+n_{A} \geqslant 12$ and/or $n_{k}+n_{A} \geqslant 8$, we take again the integral $J_{\mu \nu}^{\mathrm{lb}}(D)$ in Eq. (B.12). Using Eq. (2.16), Eq. (B.13) and the $m$-theorem, it is very easy to see that

$$
I_{\mu \nu}^{1 \mathrm{~b}}(D)=\sum_{j=1}^{J} \alpha_{j} \frac{g^{2} c_{V}}{\mu^{D-4}} \int \frac{\mathrm{d}^{D} q}{(2 \pi)^{D}}\left[\frac{q^{4} p_{\mu} q_{\nu}}{\left(q^{4}+\Lambda^{4}\right)^{2}}-\frac{3 q^{2}(p q)\left(2 q^{4}+\Lambda^{4}\right) p_{\mu} q_{\nu}}{\left(q^{4}+\Lambda^{4}\right)^{3}}\right]+\ldots
$$

with the dots collecting integrals finite by power counting at $D=4$ that at $D=4$ vanish as $A \rightarrow \infty$ and $M_{j}$ is kept fixed. To perform the integration over $q^{\mu}$, we employ Eq. (B.27) below and obtain

$$
I_{\mu \nu}^{\mathrm{lb}}(D)=\frac{3}{2} \frac{g^{2} c_{V}}{16 \pi^{2}} \sum_{j=1}^{J} \alpha_{j} L\left(A^{2}\right) p_{\mu} p_{\nu}+v_{0}
$$

Proceeding in this way for all integrals (B.10) from diagram (1b) that escape the cut (B.18) and putting together all contributions, we end up with

$$
\begin{aligned}
(1 \mathrm{~b})= & \frac{g^{2} c_{V}}{16 \pi^{2}} \sum_{j=1}^{J} \alpha_{j}\left\{\left[\frac{3}{4} \ln \left(\frac{p^{2}}{\Lambda^{2}}\right)-\frac{9}{4} \ln \left(\frac{\Lambda^{2}}{M_{j}^{2}}\right)-\frac{15}{2}\right] M_{j}^{2} g_{\mu \nu}\right. \\
& \left.-\left[\frac{11}{4} L\left(\Lambda^{2}\right)-\frac{25}{12} \ln \left(\frac{\Lambda^{2}}{M_{j}^{2}}\right)\right] p^{2} g_{\mu \nu}-\left[13 L\left(\Lambda^{2}\right)+\frac{7}{3} \ln \left(\frac{\Lambda^{2}}{M_{j}^{2}}\right)\right] p_{\mu} p_{\nu}\right\}+v_{0} .
\end{aligned}
$$

For the other diagrams we get

$$
\begin{aligned}
& (1 \mathrm{~d})=\frac{g^{2} c_{V}}{16 \pi^{2}} \sum_{j=1}^{J} \alpha_{j}\left\{\left[\frac{9}{4} \ln \left(\frac{A^{2}}{M_{j}^{2}}\right)+\frac{15}{2}\right] M_{j}^{2} g_{\mu \nu}+\frac{4}{3} L\left(\Lambda^{2}\right)\left(11 p^{2} g_{\mu \nu}+p_{\mu} p_{\nu}\right)\right\}+v_{0} \\
& (2 \mathrm{a})=r^{2} \frac{g^{2} c_{V}}{16 \pi^{2}} \sum_{j=1}^{J} \alpha_{j}\left[\frac{3}{4} \ln \left(\frac{p^{2}}{\Lambda^{2}}\right) M_{j}^{2} g_{\mu \nu}+\frac{1}{3} \ln \left(\frac{p^{2}}{M_{j}^{2}}\right)\left(\frac{1}{4} p^{2} g_{\mu \nu}-p_{\mu} p_{\nu}\right)\right]+v_{0}, \\
& (2 \mathrm{c})+(2 \mathrm{~d})=r^{2} \frac{g^{2} c_{V}}{16 \pi^{2}} \sum_{j=1}^{J} \alpha_{j}\left\{-\frac{3}{2} \ln \left(\frac{p^{2}}{\Lambda^{2}}\right) M_{j}^{2} g_{\mu \nu},\right.
\end{aligned}
$$




$$
\left.+\frac{1}{6}\left[L\left(\Lambda^{2}\right)+8 \ln \left(\frac{\Lambda^{2}}{M_{j}^{2}}\right)\right] p^{2} g_{\mu \nu}-\frac{2}{3}\left[L\left(\Lambda^{2}\right)+\frac{5}{4} \ln \left(\frac{\Lambda^{2}}{M_{j}^{2}}\right)\right] p_{\mu} p_{\nu}\right\}+v_{0} .
$$

Eq. (4.10) follows from Eqs. (B.4)-(B.9) and (B.19)-(B.22). Note again that the sums $\sigma_{l}$ are finite and that the resulting vacuum polarization tensor is transverse.

Case $0<\theta_{j}<\infty$

Using the same type of arguments methods as for the previous two cases, we have

$$
\begin{aligned}
& \text { (1b) }=\frac{g^{2} c_{V}}{16 \pi^{2}} \sum_{j=1}^{J} \alpha_{j}\left[a_{1} \Lambda^{2} g_{\mu \nu}-L\left(\Lambda^{2}\right)\left(\frac{11}{4} p^{2} g_{\mu \nu}+13 p_{\mu} p_{\nu}\right)\right]+v_{0} \\
& (1 \mathrm{~d})=\frac{g^{2} c_{V}}{16 \pi^{2}} \sum_{j=1}^{J} \alpha_{j}\left[a_{2} \Lambda^{2} g_{\mu \nu}+\frac{4}{3} L\left(\Lambda^{2}\right)\left(11 p^{2} g_{\mu \nu}+p_{\mu} p_{\nu}\right)\right]+v_{0} \\
& (2 \mathrm{a})=r^{2} \frac{g^{2} c_{V}}{16 \pi^{2}} \sum_{j=1}^{J} \alpha_{j}\left[a_{3} \Lambda^{2} g_{\mu \nu}+\frac{1}{3} \ln \left(\frac{p^{2}}{\Lambda^{2}}\right)\left(\frac{1}{4} p^{2} g_{\mu \nu}-p_{\mu} p_{\nu}\right)\right]+v_{0}, \\
& (2 \mathrm{c})+(2 \mathrm{~d})=r^{2} \frac{g^{2} c_{V}}{16 \pi^{2}} \sum_{j=1}^{J} \alpha_{j}\left[a_{4} \Lambda^{2} g_{\mu \nu}+\frac{1}{3} L\left(\Lambda^{2}\right)\left(\frac{1}{2} p^{2} g_{\mu \nu}-2 p_{\mu} p_{\nu}\right)\right]+v_{0} .
\end{aligned}
$$

Here $a_{1}, \ldots, a_{4}$ are coefficients that depend on $\theta_{j}$ and that can be expressed in terms of the roots in the complex upper half-plane of the polynomial $P(z)=z^{6}+z^{2}+\theta_{j}^{2}$.

\section{Useful integrals.}

We finish this appendix by giving the following two dimensionally regularized integrals that have been widely used in the computations and that are not in the tables:

$$
\frac{1}{\mu^{D-4}} \int \frac{\mathrm{d}^{D} q}{(2 \pi)^{D}} \frac{\left(q^{2}\right)^{\eta}}{\left(q^{4}+\lambda^{4}\right)^{\gamma}}=\frac{\lambda^{4+2 \eta-4 \gamma}}{32 \pi^{2}}\left(\frac{\lambda^{2}}{4 \pi \mu^{2}}\right)^{\frac{p}{2}-2} \frac{\Gamma\left(\frac{D}{4}+\frac{\eta}{2}\right) \Gamma\left(\gamma-\frac{D}{4}-\frac{\eta}{2}\right)}{\Gamma(\gamma) \Gamma\left(\frac{D}{2}\right)},
$$

$\frac{1}{\mu^{D-4}} \int \frac{\mathrm{d}^{D} q}{(2 \pi)^{D}} \frac{\left(q^{2}\right)^{\eta}}{\left(q^{6}+\lambda^{6}\right)^{\gamma}}=\frac{\lambda^{4+2 \eta-6 \gamma}}{48 \pi^{2}}\left(\frac{\lambda^{2}}{4 \pi \mu^{2}}\right)^{\frac{D}{2}-2} \frac{\Gamma\left(\frac{D}{6}+\frac{\eta}{3}\right) \Gamma\left(\gamma-\frac{D}{6}-\frac{\eta}{3}\right)}{\Gamma(\gamma) \Gamma\left(\frac{D}{2}\right)}$.

\section{References}

[1] G. 't Hooft and M. Veltman, Nucl Phys. B 44 (1972) 189.

[2] A.A. Slavnov, Theor. Math. Phys. 33 (1977) 977.

[3] L.D. Faddeev and A.A. Slavnov, Gauge fields, introduction to quantum theory, second edition (Benjamin, Reading, 1990). 
[4] Z. Bern, M.B. Halpern, L. Sadun and C. Taubes, Nucl. Phys. B 284 (1987) 35.

[5] M. Asorey and F. Falceto, Nucl. Phys. B 327 (1989) 427.

[6] A.A. Slavnov, Symmetry preserving regularization for gauge and supergauge theories, in Superspace and supergravity, ed. S.W. Hawking and M. Rocek (Cambridge Univ. Press, Cambridge, 1981 ) p. 177.

[7] B.J. Warr, Ann. Phys. 183 (1988) 1.

[8] R. Sénéor, Some remarks for the construction of Yang-Mills theories, in Renormalization of quantum field theories with non-linear field transformations, ed. P. Breitenlohner, D. Maison and K. Sibold (Springer-Verlag, Berlin, 1988).

[9] M. Day, Nucl. Phys. B 213 (1983) 591.

[10] REDUCE 3.4.1 and 3.5 (The Brand Corporation, Santa Monica, 1991 and 1993).

[11] O. Piguet and A. Rouet, Phys. Rep. 76 (1981) 1.

[12] C.P. Martin and F. Ruiz Ruiz, Higher covariant derivative regulators and non-multiplicative renormalization, NIKHEF-H preprint, to appear in Phys. Lett. B.

[13] G. 't Hooft and M. Veltman, Diagrammar, in Particle interactions at very high energies, ed. D. Speiser, F. Halzen and J. Weyers (Plenum Press, London, 1974).

[14] E.H. Lifshitz and L.P. Pitaevskii, Relativistic quantum theory, Part 2, Chapters XI and XII (Pergamon Press, New York, 1973).

[15] H. van Dam and M. Veltman, Nucl. Phys. B 22 (1970) 397.

[16] G. Giavarini, C.P. Martin and F. Ruiz Ruiz, Nucl. Phys. B 381 (1992) 222.

[17] J.C. Collins, Renormalization (Cambridge Univ. Press, Cambridge, 1987). 